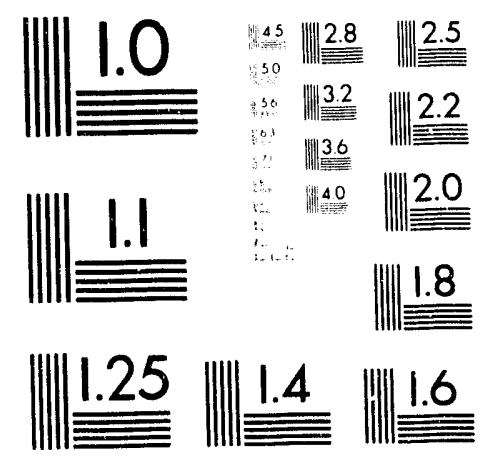



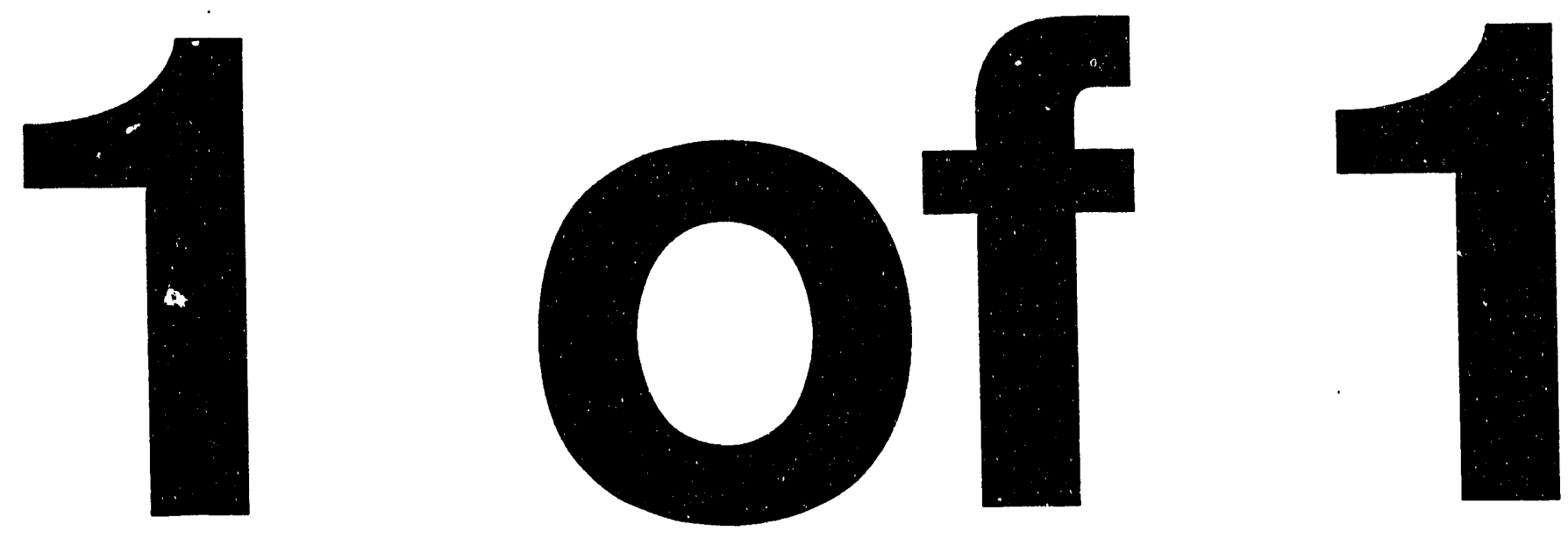


\section{The Relativistic Heavy Ion Collider Facility}

June 1993

Prepared by

T.W. Ludlam and A.J. Stevens

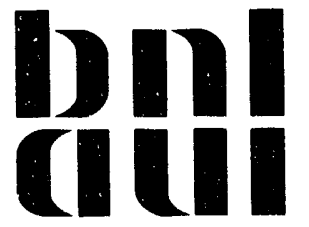

$\operatorname{SeP} 10103$

$0 S T I$

BROOKHAVEN NATIONAL LABORATORY ASSOCIATED UNIVERSITIES, INC.

UPTON, NEW YORK 11973

UNDER CONTRACT NO. DE-AC02-76CHOOO16

UNITED States department of EneRgy 


\section{DISCLAIMER}

This report was prepared as an account of work sponsored by an agency of the United States Government. Nrither the United States Government nor any agency thereof, nor any of their employees, nor any of their contractors, subcontractors, or their employees, makes an: warranty, express or implied, or assumes any legal liability or responsibility for the accuracy, completeness, or usefulness of any information, apparatus. product, or process disclosed, or represents that its use would not infringe privately owned rights. Reference herein to any specific commercial product, process, or service by trade name, trademark, manufacturer, or otherwise, does not necessarily constitute or imply its endorsement, recommendation, or favoring by the United States Government or any agency, contractor or subcontractor thereof. The views and opinions of authors expressed herein do not necessarily state or reflect those of the United States Government or any agency, contractor or subcontractor thereof. 


\section{Table of Contents}

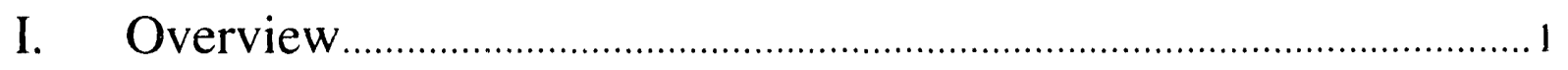

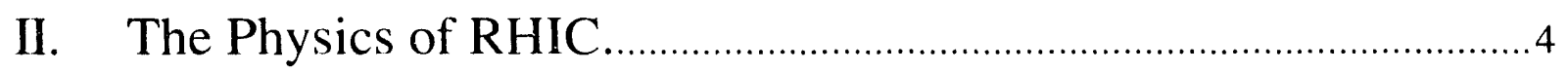

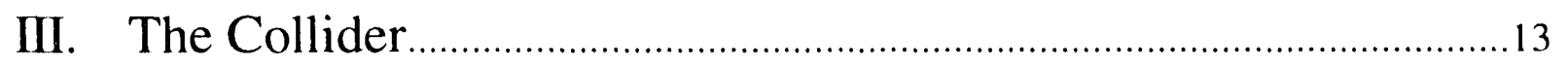

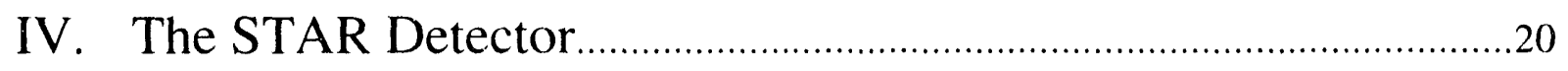

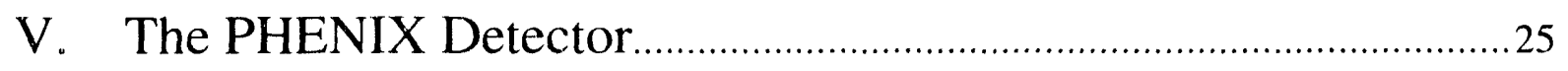

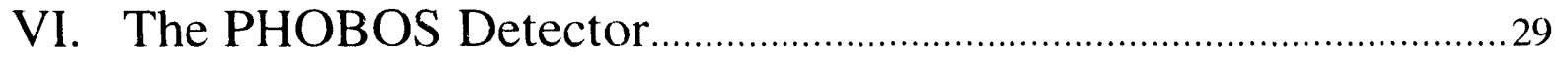

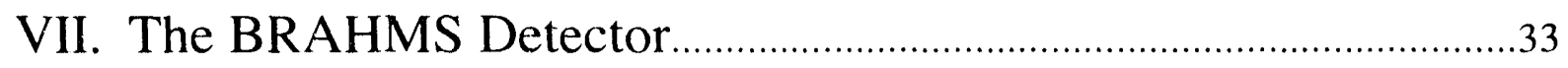




\section{Overview}

With the realization, some twenty years ago, that the "elementary" particles in nature have a substructure of quarks, speculation began on the possibility of creating a form of matter in which the recognizable components are not the familiar neutrons and protons, but are quarks and the gluons through which they interact. The development of Quantum Chromodynamics (QCD) as a fundamental theory of strong interactions provided firm predictions that at extremely high (but apparently achievable) values of temperature and density, ordinary nuclear matter can be transformed into a plasma of quarks and gluons. An international community has since developed among nuclear and particle physicists with a common interest in the possibility of creating and studying this new state of matter by colliding heavy nuclei at extremely high energies.

With RHIC, the United States Department of Energy is constructing a world-class scientific facility to open up this new frontier, and with it an unparalleled potential for new discovery in the realm of Nuclear Physics. RHIC will be the first accelerator to collide beams of heavy nuclei at high eneigies. By doing so, RHIC will create a microcosm of the hot, dense plasma of quarks and gluons from which elementary particles as we know them are thought to have condensed, immediately after the "Big Bang" of the early universe.

By means of extraordinarily high energy nuclear collisions, RHIC will act as a giant pressure cooker that will produce temperatures and densities more extreme than exist row even at the centers of stars--creating new states of matter and opening the way to a new understanding of the relationship between the fundarnental constituents of matter and the complex array of particles and nuclei that make up our universe.

The RHIC collider will consist of two beams of heavy ions circulating in opposite directions around a pair of superconducting magnet rings in a tunnel 3.8 kilometers in circumference. Where the beams collide at six different points around the accelerator, experiments will be carried out as interactions occur with an energy of 100 billion volts for each of the protons and neutrons contained in the colliding nuclei $(100 \mathrm{GeV} / \mathrm{u})$. Cillisions that combine heavy nuclei and extremely high energies are the key to creating a quark-gluon plasma. This cannot be done at existing high energy accelerator facilities.

The completed RHIC facility will be a complex array of accelerators, utilizing existing $\mathrm{BNL}$ machines to pre-accelerate and inject ions into the collider rings. 


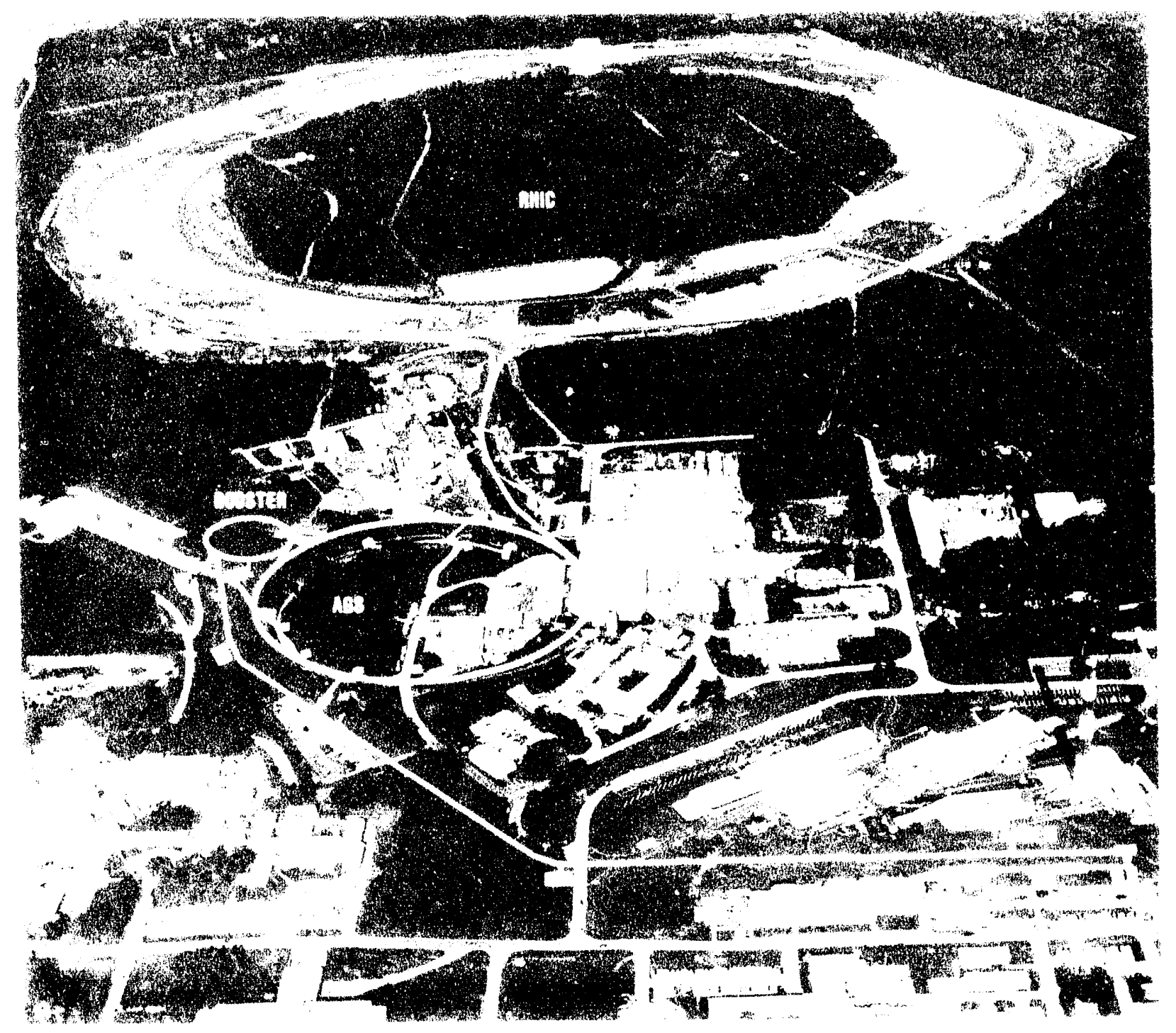


around the rings in stable orbits for hours. For experiments, at the intersection points where the beams cross, particles will collide at the rate of tens of thousands of collisions per second.

Formal construction began in January, 1991, after the US. Congress appropriated the first construction funds for RHIC. The total cost of construction will be about $\$ 480$ Millicn, of which approximately one quarter will be devoted to building the initial complement of detectors. The collider will be ready for experiments by early 1999.

A major part of the construction effort for RHIC is the production of the superconducting magnets that will guide the beams around the rings. RHIC will use about 1700 superconducting magnets. Brookhaven has developed and tested prototypes of each type of magnet needed for RHIC. With a few exceptions, the final magnets will be built by industry. In 1992, contracts to build superconducting dipole and quadrupole magnets for RHIC were awarded to Grumman Aerospace Corporation, and the technology transfer from BNL to Grumman for the production manufacture of these magnets commenced in early 1993.

As the machine construction has gotten underway, a parallel effort is well along to define the initial research program and begin construction of the first round of detectors. An international scientific community, involving 6 US. national laboratories, approximately 30 US. universities, and over 10 foreign countries has begun building two large detectors for RHIC experiments. These detectors, called STAR and PHENIX, will be complemented by a number of smaller experiments, some of which (BRAHMS and PHOBOS) are well into the design stage, and some of which have yet to be proposed.

The remaining sections of this document briefly describe the physics of RHIC, the collider, and four of the detectors which are now planned to explore the new realm of high energy heavy ion physics.

Thomas W. Ludlam

Associate Head, RHIC Project

Detectors and Experiment Support

June, 1993 


\section{The Physics of RHIC}

The essential motivation for colliding heavy nuclei at ultra relativistic energies is the production of strongly interacting matter at extreme conditions of temperature and density. Extended volumes of hadronic matter with energy densities greater than 10 times that of the nuclear ground state should be realizable, with initial temperatures that far exceed the so-called "limiting temperature" (Hagedorn temperature) observed for mesons emitted in high energy hadron collisions. The extreme conditions produced in a heavy ion system are expected to last long enough for the constituents (quarks or hadrons) to approach local thermal equilibrium. The lifetime of this system is at least the radius of the colliding nuclei ( $7 \mathrm{fm}$ for $\mathrm{Au}$ ) divided by the speed of light, but may be much longer. By using the largest projectiles available, one therefore has the best chance of producing a long lived thermal system. There is little direct knowledge about what to expect under such conditions. They have not been detected anywhere in the natural universe, and are just beginning to be approached through experiments with high energy ion beams in fixed targets at Brookhaven and CERN. It is believed that under such extreme conditions the basic structure of hadronic matter should undergo a fundamental change - a phase transition - from a system of nucleons and mesons to one of unconfined quarks and gluons.

Our knowledge of the strong interaction has developed from the study of high energy collisions of elementary particles, and is embodied in the theory of Quantum Chromodynamics (QCD). The theory predicts that it is possible to assemble macroscopic volumes of nuclear matter at such extreme thermodynamic conditions as to overcome the forces that bind quark -antiquark or three-quark systems, leading to a phase transition from ordinary hadronic matter to a quark-gluon plasma.

The goal of RHIC experiments is to confirm this prediction of a deconfined high-energy-density phase of matter, to search for the quark-gluon plasma, and study the properties of this new state of matter. More generally, the goal is to determine the thermodynamic properties of the states produced in high energy nuclear collisions, and to understand the phase structure as these states evolve from the initial collision to the final burst of particles that reaches the detectors.

Central to the predicted phase structure of QCD thermodynamics is the phenomenon of chiralsymmetry restoration. At high densities, the deconfined quarks undergo a shift in their effective mass from the normal value of about $300 \mathrm{MeV}$ to the zero-mass quarks of the ideal "chiral symmetric" QCD equations of motion. The chiral symmetry is spontaneously broken in normal matter, where 
quarks are confined in hadrons. In the deconfined phase chiral symmetry is restored, with corresponding changes in the dynamics of interacting quarks.

Strongly interacting matter can achieve high density by virtue of very high temperatures (i.e. through the creation of quark-antiquark pairs as the vacuum is heated), or by extreme compression of nuclear matter at lower temperatures. Both of these conditions can be reached in high energy heavy ion collisions.

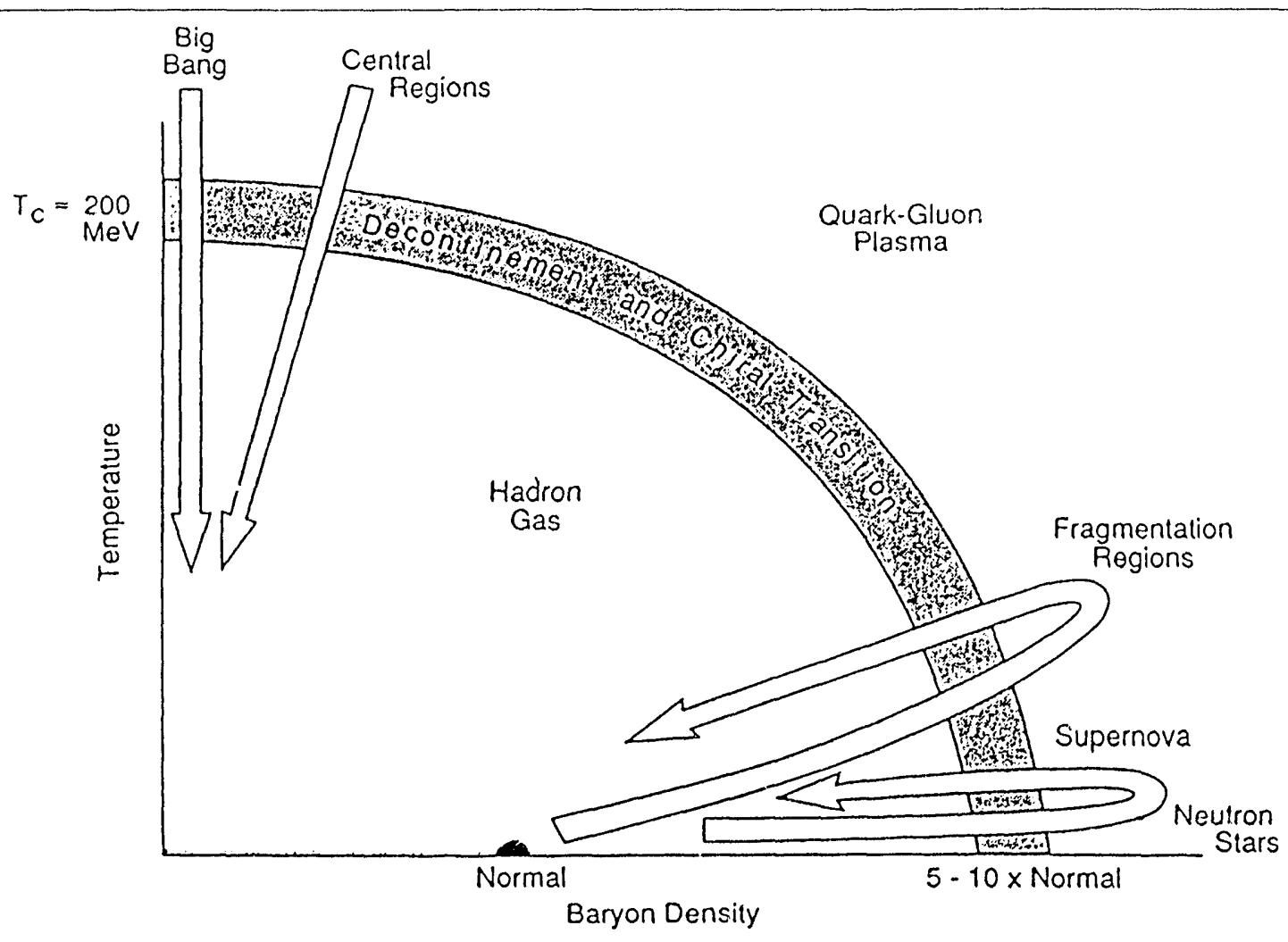

phase diagram of nuclear matter. Temperature is plotted vs. net baryon clensity for an extended volume of nuclear matter in thermal equilibrium. Normal nuclear matter appears at the point shown on the density axis, at zero temperature: this is the neighborhood explored by traditional nuclear physics experiments. The region of the phase transitions corresponding to quark deconfinement (at temperature $\mathrm{T}_{\mathrm{C}}$ ) and chiral symmetry restoration is indicated. Above $\mathrm{T}_{\mathrm{C}}$, hadrons dissolve into quarks and gluons. Above the temperature of chiral symmetry restoration quarks are massless. The two transition temperatures may well be coincident. The indicated trajectories show two avenues for probing the quark-gluon plasma with high energy nucleus-nucleus collisions: by reaching high baryon densities among the hot, compressed fragments of the colliding nuclei, and at very high temperatures in the central rapidity region among thermally produced particles where conditions may approximate those of the early universe.

Using the calculational techniques of lattice gauge theory, the dynamics of QCD can be incorporated in statistical calculations to derive the resulting thermodynamics of macroscopic, strongly 
interacting systems. Such calculations predict the phase transition to deconfined quark matter. For zero baryon density, the critical temperature for this transition is about $150-200 \mathrm{MeV}$.

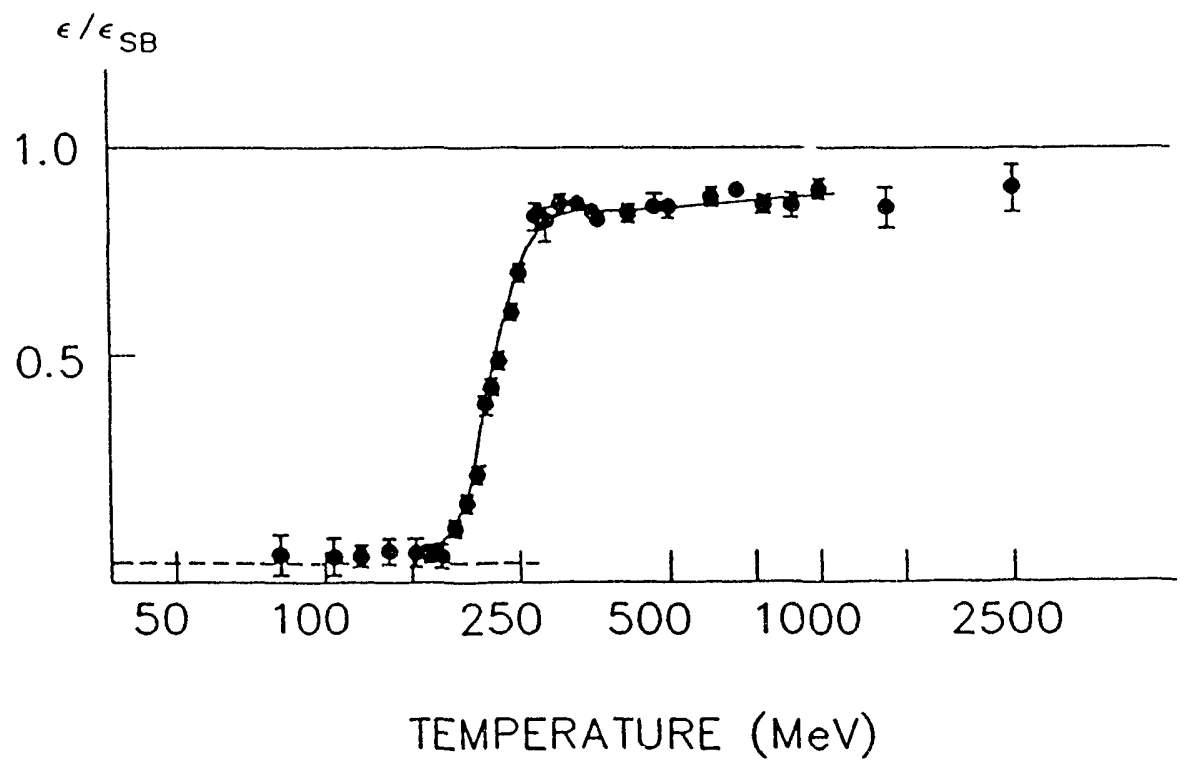

phase transition in lattice gauge calculation. The generic behavior predicted by these calculations is shown in an early QCD lattice result, in which the energy density is shown as a function of the temperature. Monte Carlo techniques are used to evaluate the partition function on a finite space-time lattice, hence the calculation appears as discrete points with statistical errors. Here, the energy density $\varepsilon$ is normalized to the ideal Stephan-Boltzmann gas form $\varepsilon_{S B}$. At a critical temperature $T_{C}$, an abrupt change occurs corresponding to a first-order phase transition from an ideal hadron gas to an ideal quark-gluon plasma. Present lattice QCD calculations are probing the details of particle structure and thermodynamic properties near the phase transition, and attempting to extend the results to the case of non-zero baryon density. While the order of the physical phase transition is still uncertain, depending delicately on the effective masses of the quarks, the critical energy density for plasma formation is clearly predicted to be in the range $1-3 \mathrm{GeV} / \mathrm{fm}^{3}$. This is to be compared with an energy density of $0.15 \mathrm{GeV} / \mathrm{fm}^{3}$ for normal nuclear matter, and a density of $0.5 \mathrm{GeV} / \mathrm{fm}^{3}$ inside a nucleon.

The remarkable fact is that this fundamental change of phase of hadronic matter should occur at nuclear densities only about one order of magnitude greater than that of normal nuclear matter, at energy densities of a few $\mathrm{GeV} / \mathrm{fm}^{3}$. Such high energy densities are conjectured to have prevailed in the very early universe, until 5-10 microseconds after the Big Bang. They may exist today within the cores of neutron stars. The possibility of creating these conditions for investigation in the laboratory is one of the great challenges for modern experimental physics.

\section{Characteristics of High Energy Nucleus-Nucleus Collisions}

The belief that this thermodynamic environment is achievable in high energy nuclear collisions is based on the observed particle production in high energy proton-proton and proton-nucleus collisions, 
and on data from multi-GeV fixed target collision with nuclear beams that are now available at the Brookhaven AGS and CERN SPS accelerators.

These experiments have established that states of compressed nuclear matter can be created and studied under laboratory conditions in which extreme values of energy density are achieved. The data presently under investigation reflect an environment of high baryon density, at collision energies where the impinging nuclei are nearly stopped in the collision. Further extrapolation is required to predict the behavior at much higher energies, the collider regime, at which the colliding nuclei fly through the collision volume without stopping, leaving behind a hot, dense "central region" with near-zero baryon number in which particles are produced quite literally by heating the vacuum.

For very high energy collisions, particle spectra are often displayed in terms of a useful longitudinal scaling variable called rupidity $(\mathrm{y})$ :

$$
y=\frac{1}{2} \ln \left(\frac{E+p_{l}}{E-p_{l}}\right)
$$

where $\mathrm{E}$ is the particle energy and $p_{t}$, the longitudinal component of momentum. In high energy $p-p$ and $\bar{p}-p$ collisions, the rapidity spectrum reveals a characteristic pattern of particle production: for c.m. collision energy $\sqrt{S}$ GeV the available rapidity interval is $\pm y_{\text {max }}^{\prime}$, where $y_{\text {max }} \equiv \ln \sqrt{S}$. For collisions with $y_{\operatorname{mix}} \geq 3$ units, the rapidity density exhibits a plateau near $y=0$, with the net baryon number (baryons minus anti baryons) constituting a small fraction of the total particle density. This is the so-called "central region" of rapidity. The baryons are mainly confined to the "fragmentation" regions, which lic within 1.5 2 units of the end-points of the spectrum:

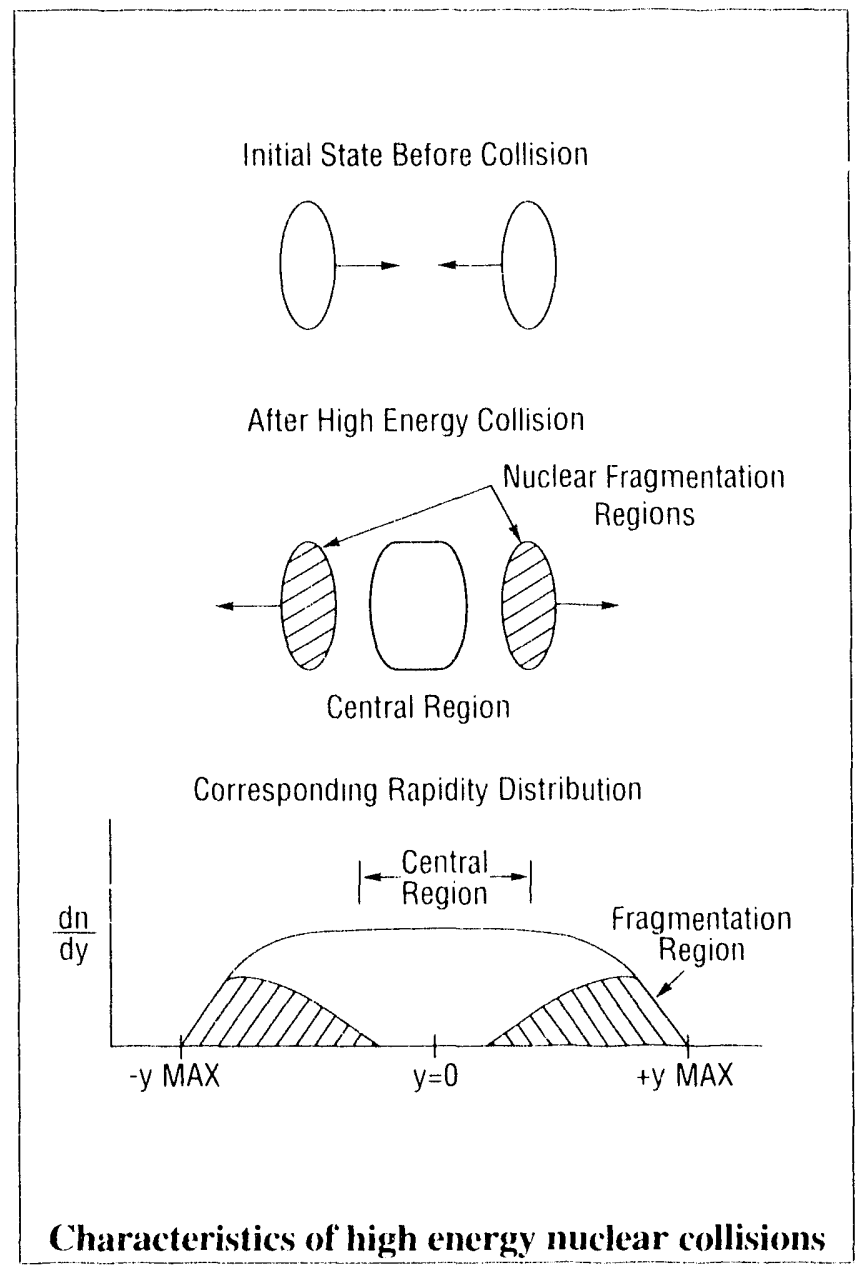

The energy dependence of the main features of particle production in hadron-hadron collisions are well established: as the collision energy is increased the width of the central region grows logarithmically with energy; the particle density in the fragmentation region remains fixed; and the 
density of particles at $y=0$ grows slowly (logarithmically) with energy. The top energy of RHIC $\left(\sqrt{S} / u=200 G e V, v_{\max }=5.3\right)$ is well into this high energy regime.

For $p-p$ and $\bar{p}-p$ collisions the average multiplicity per unit central rapidity is well approximated by the form $(d n / d y)_{v=10} \equiv 0.8 \ln \sqrt{S}$. For these single hadron collisions, the density of particles at central rapidity is thus approximately 4 per rapidity unit at the RHIC collision energy of 200 $\mathrm{GeV}$.

The appearance of a rapidity plateau at high energies corresponds to a scaling behavior in which the thermodynamics of the system are the same within each interval of rapidity. In this domain the energy density can be expressed in terms of the total energy carried off by the secondary particles in one unit of $y$, and the initial volume in which these particles are formed:

$$
V_{0}=\pi R_{A}^{2} \tau_{0}
$$

where $R_{\mathrm{A}}$ is the nucleon radius and $\tau_{0}$ is the particle formation time, roughly $1 \mathrm{fm} / \mathrm{c}$. Thus:

$$
\begin{aligned}
& \varepsilon\left(G e V / \mathrm{fm}^{3}\right)=\frac{1}{V_{0}}\langle E\rangle \frac{d n}{d y} \\
& \langle E\rangle=\text { energy per particle } \approx 0.5 \mathrm{GeV} .
\end{aligned}
$$

For nuclear collisions the multiplicity density $d n / d y$ scales as $A^{\alpha}$, where the value of $\alpha$ derived from proton-nucleus collisions is approximately 1.1 .

Extrapolating to collisions of gold ions at RHIC then, we find an environment of unprecedented extremes:

- The estimated particle densities exceed 1000 per unit rapidity, with many thousands of particles emitted per collision.

- The initial energy density in such collisions should be in the range $5-10 \mathrm{GeV} / \mathrm{fm}^{3}$. Thus, initially, the average density over a volume comparable to a gold nucleus should exceed normal nuclear matter density by nearly two orders of magnitude, and may exceed by a factor of 10 or more the normal density inside a proton.

\section{Experiments and Detectors at RHIC}

The term "mini-bang" has been used to describe the interactions to be explored at RHIC, as each collision unfolds a sequence of events that calls to mind the formation of matter in the evolution of 
the cosmos. In the current thinking, this sequence of events is as follows: After a formation time $\left(\tau_{0)}\right)$ of about $1 \mathrm{fm} / \mathrm{c}$. the system (roughly the size of the colliding nuclei) becomes thermalized in a plasma of quarks and gluons. This system then expands and coois, and when it reaches the critical temperature of the phase transition, hadrons begin to form. After that it becomes a gas of interacting hadrons, possibly in thermal equilibrium. As expansion continues a freeze-out density is reached at which the hadrons no longer interact with each other. The detected particles are those that emerge from this freeze-out volume.

Thus, experiments at RHIC are in many ways analogous to certain astronomical observations. The object of interest is an extended source emitting copious radiation whose spectra may reflect thermal. and possibly chemical equilibrium of the radiating source. As with stellar observations, different types of radiation may carry signals characteristic of distinct stages as the system evolves in space and time.

In the case of the nuclear collisions at RHIC. most of the many thousands of particles emitted are hadrons produced at the surface of the interacting volume. They provide information about the size, the thermal properties. and the relative abundance of particle species at freeze-out. Information from the hot interior of the expanding volume can only be carried to the outside by radiation that is not coupled to the strong interaction. i.e. not hadrons. Such signals can be carried by photons, which may be "real" photons (gamma rays) or "virtual" photons (appearing as electron-positron or muon-antimuon pairs). These direct. electromagnetic signals are much les, abundant than the hadrons, comprising about .01\% of all the particles emerging from the collision, and require very specialized instruments to resolve them from the hadronic background.

The RHIC detectors are specially designed and finely tuned for this environment. They are capable of measuring very high densities of relatively "soft" (i.e. low-momentum) particles with precise determination of the kinematic properties and quantum numbers of each particle within selected solid angles. Because of the thermal nature of the interactions. RHIC detectors are not designed to reconstruct the entire event over all angles (i.e., to be "hermetic"). as is generally the case at other high energy colliders.

For the initial research at RHIC, an array of complementary detectors will provide specialized instrumentation to explore all of these facets of the predicted behavior of a quark-gluon plasma, and set as wide a net as possible for unexpected discoveries. 


\section{Hadronic measurements: the global picture}

The main features of these high energy nuclear collisions are reflected in the abundance of hadrons produced. The total multiplicity of particles in a given interval of rapidity, and the flow of energy in the transverse and longitudinal directions after the collision, serve to categorize the degree of thermalization and the extent to which the interacting nuclear matter has been excited to a state of elevated energy density. Such measurements are often referred to as global variables, and are used as basic triggers for the RHIC detectors.

A large solid angle detector capable of reconstructing thousands of particles in a single event-such as the STAR detector-- will focus in much more detail on hadrons, the "surface radiation", and will measure the temperature and degree of thermalization of the expanding matter at freeze-out. Measurements of the relative abundance of strange particles, baryons, hyperons, and their antiparticles, will serve to establish the thermodynamic properties and the quark chemistry of the evolving system and to help determine the conditions under which the primordial plasma was formed. These experiments will provide the kind of global survey needed to guide the early research at RHIC.

Data on high multiplicity hadron production in these collisions leads to a unique and powerful new class of measurements: the determination of space-time dimensions of radiating sources through like-particle interferometry. This is a generalization of the Hanbury-Brown Twiss (HBT) method used to measure the size of distant stars. from a study of the momentum correlations among identical hadrons it will be possible to determine the extent of the volumes in space and time from which various types of particles (e.g. pions, kaons, protons) are emitted. Multiparticle interference phenomena may also result in large scale coherent effects triggered by extremely high particle density. Speculation includes the possibility of stimulated emission of particles (e.g. pion lasers), and anomalous fluctuations in the relative number of charged and neutral particles, similar to the CENTAURO class of cosmic ray events.

Entirely new forms of hadronic matter may be observed as a consequence of quark-gluon plasma formation. If such a plasma provides an initial system with equal numbers of up, down and strange quarks, as may be the case in a high-temperature plasma of quark matter, many calculations predict the formation of strange matter, or strangelets upon freeze-out: stable or metastable droplets of nuclear matter with high baryon number $(\approx 10)$ or greater), and near-zero electric charge because of the presence of numerous strange quarks. 
Radiation from a "Mini-Bang":

Signals Probe Different Space-time Volumes

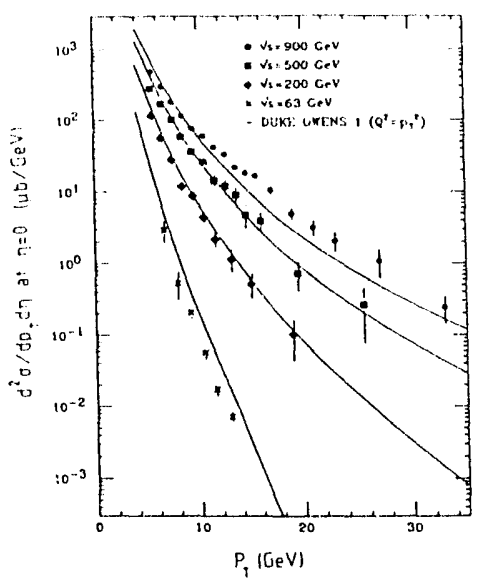

QCD Jets

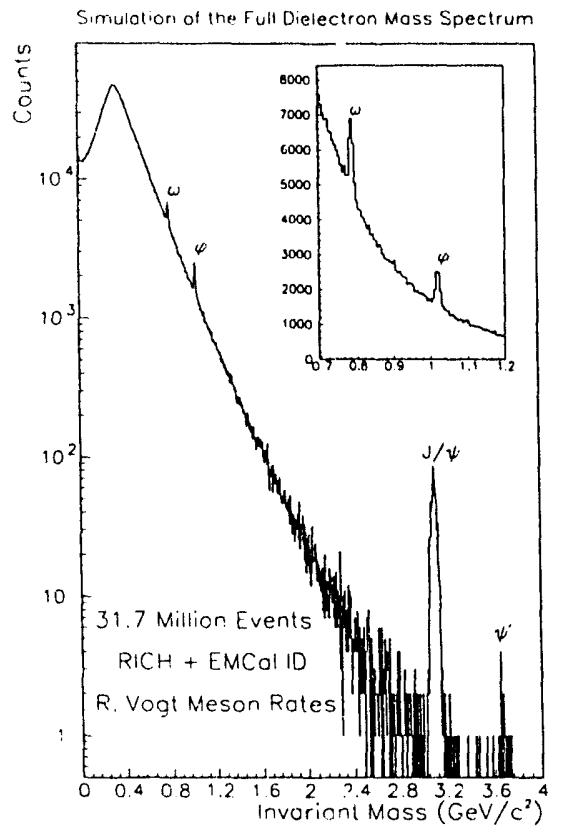

Fhotons \& Lepton Pairs

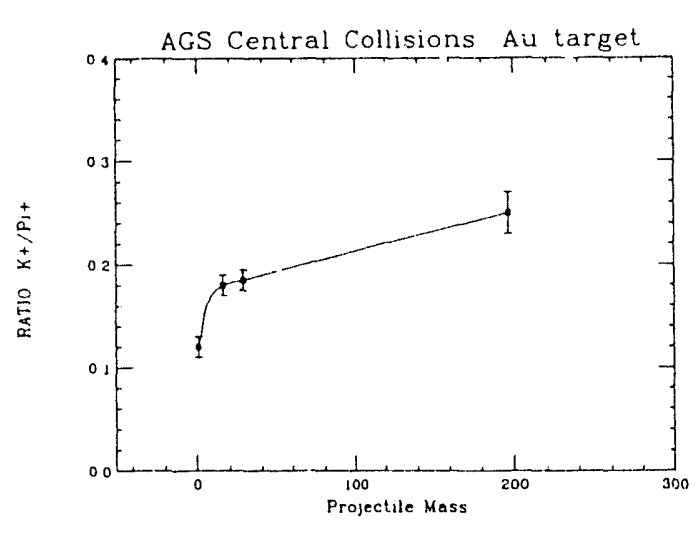

Strange Particles

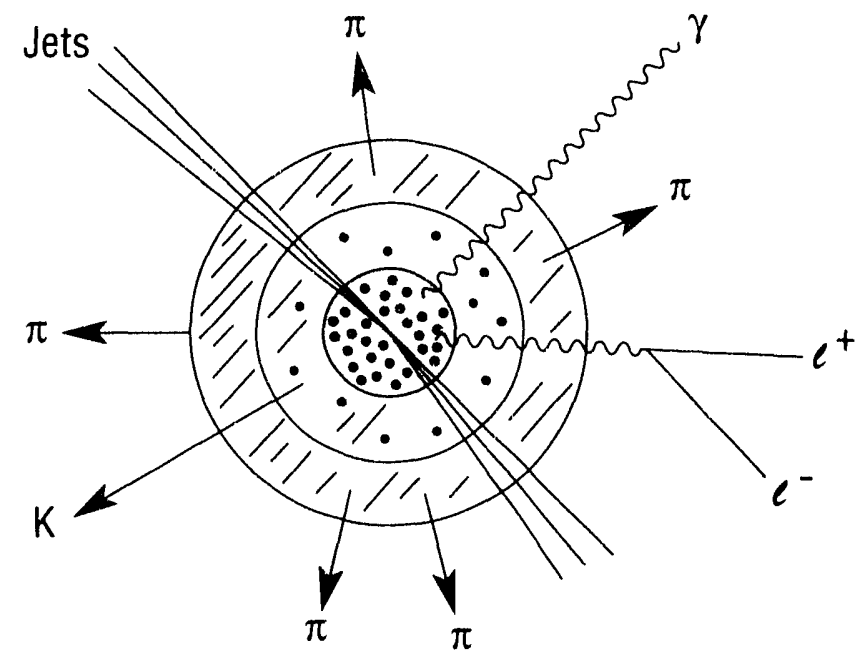

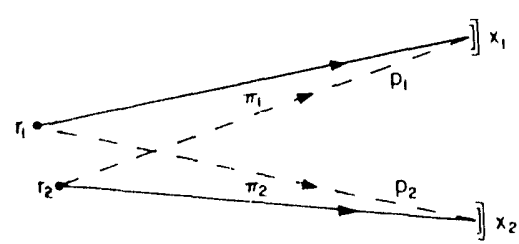

Particle Interferometry

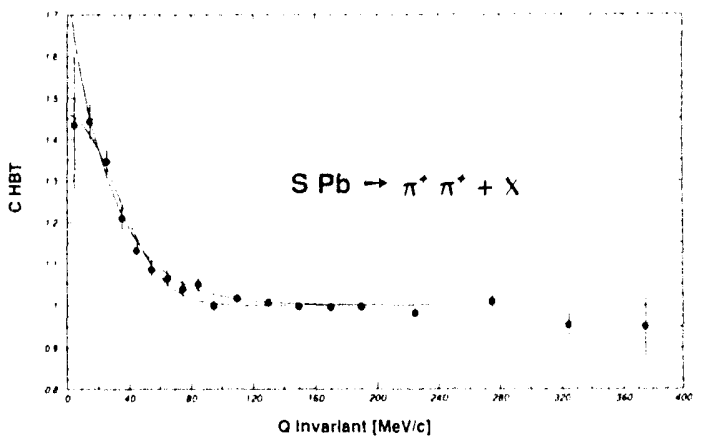




\section{Leptons, photons and jets: probing the early phase}

The energy spectrum of photons radiated directly from the initial plasma state can provide information about the degree of thermalization, and the temperature of the plasma. The mass spectra of lepton pairs produced directly in the plasma can also serve as a "thermometer" to determine the temperature of the primordial matter.

The dilepton spectra also contain resonance signals, and to the extent that some of these resonance states were produced and then decayed in the high density phase, their properties may tell us something about the medium at this early stage. For example, changes in the effective quark masses (chiral symmetry restoration) result in changes in the predicted masses, lifetimes and branching ratios of the lighter meson resonances, $\rho, \omega, \phi$.

To determine more about the physical properties of the matter produced in the collision, it is possible to study signals produced at the very earliest stages, prior to thermalization, and look for effects due to the surrounding medium as these signals propagate through the evolving dense matter. One of the most discussed of such effects in the suppression of particle states made up of heavy quark pairs, $c \bar{c}$ or $b \bar{b}$. The primary examples of such states are the $\mathrm{J} / \psi$ and $\Upsilon$ resonances. The production of such heavy quark pairs is due to hard scattering of quarks from the incident nuclei in the first stages of the interaction. Normally, these heavy quark pairs, once produced, would bind together to form the now-familiar resonance states. It is predicted that in the presence of a quark-gluon plasma this binding will be prevented -- leading to a strong suppression of these states in collisions where a plasma is produced.

Along similar lines, the collision energy at RHIC is high enough to produce high $\mathrm{p}_{\mathrm{t}}$ hadronic jets as a result of hard quark, or gluon, scattering. These high energy quarks and gluons subsequi-ntly hadronize not in the vacuum, but in the dense medium of the plasma. The observed properties of the jets can then reflect the physical properties of the medium they have traversed.

\section{Suggested Further Reading}

E.V. Shuryak, "Quantum Chromodynamics and the Theory of Superdense Matter." Physics Reports 61.71 (1980). H. Satz. "The Transition from Hadron Matter to Quark-Gluon Plasma," Ann. Rev. Nucl. Part. Sci. $\underline{35}, 245$ (1985). Proceedings of the Ninth International Conference on Ultra-Relativistic Nucleus-Nucleus Collisions (Quark Matter '91)," T. Awes et. al.. eds., North Holland, 1992. 


\section{The Collider}

\section{A. The Facility}

The complete RHIC facility will be a complex of accelerators interconnected by beam transfer lines. The RHIC tunnel is located towards the northwest corner of the Brookhaven site. The site plan in Fig. III.1 shows all major components of the RHIC complex.

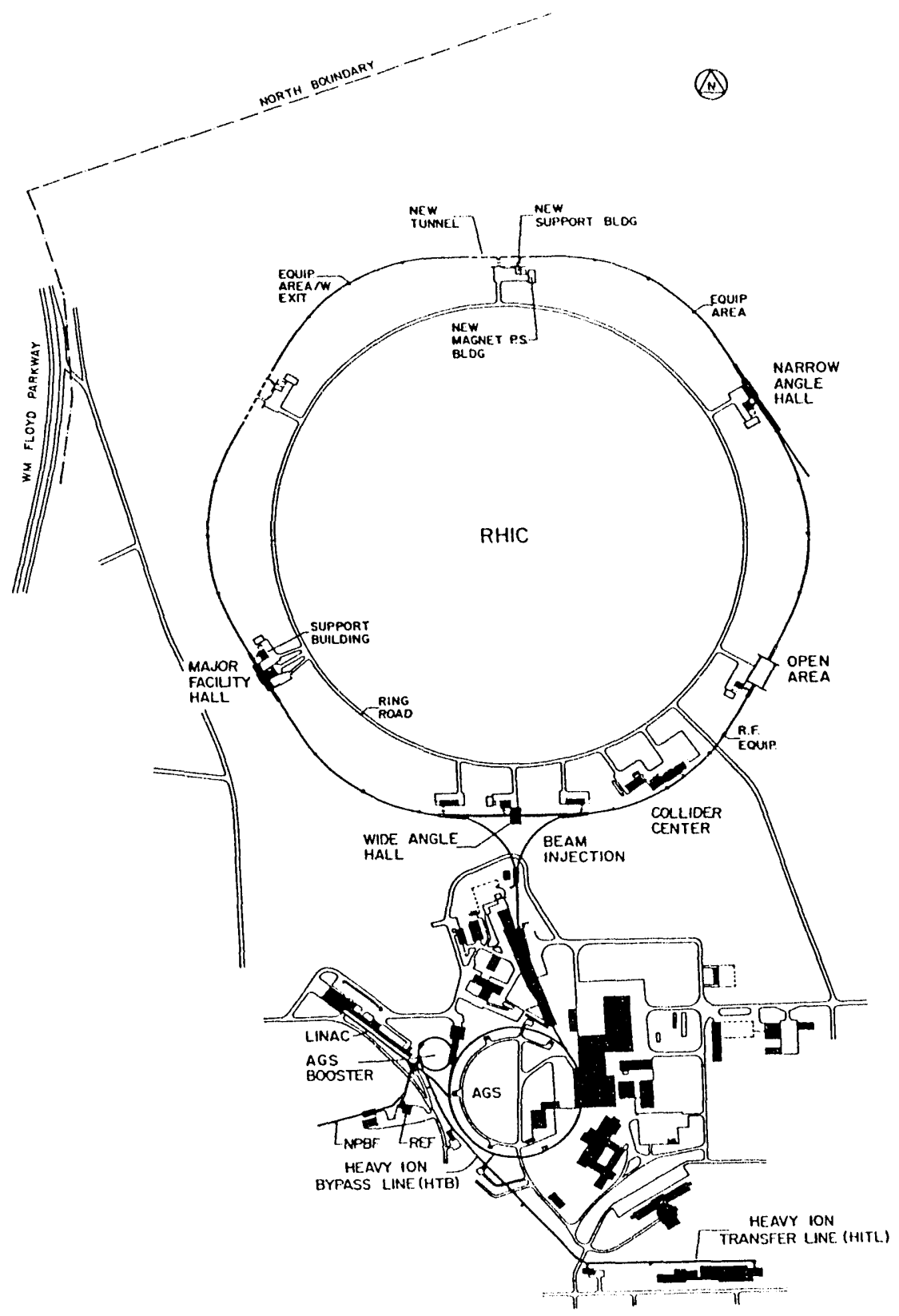

Fig. IIl.1 Layout of the RHIC Project - Collider \& Injector 
The accelerator systems included in the RHIC Project per se consist of two intersecting superconducting storage rings, the beam transfer line from the AGS (Alternating Gradient Synchrotron) to the collider rings, and the ancillary accelerator systems required for collider operation.

The collider rings are laid out in six regular "arcs" connected by "insertion regions" where the beams are tuned as they approach the collision point. The main components of the magnet system are 288 arc-size dipoles, and 108 insertion dipoles, and 276 arc and 216 insertion quadrupoles. In addition to dipoles and quadrupoles, there will be an inventory of smaller magnets consisting of 72 trim quadrupoles, 288 sextupoles, and 492 corrector magnets at each quadrupole. The cold bore beam tube was chosen to be $69 \mathrm{~mm}$ in diameter in order to accommodate the requirements due to intrabeam scattering. This is the phenomena by which particles moving together in the same bunch scatter frequently with one another because of the Coulomb repulsive forces; during the scatter the particles exchange their momenta and, in particular, longitudinal momentum can be transferred to the transverse component and vice-versa. This causes a continuous increase in the beam emittance (size) and momentum spread which must be confined within the magnet aperture and contained by the rf system. This effect is most severe for the heaviest (i.e., the most highly charged) ions

Each arc is made of eleven regular FODO cells, having the following sequence: focusing quadrupole, bending dipole, defocusing quadrupole, bending dipole. The maximum horizontal and vertical beta functions are both 49.2 meters, in the middle of the quadrupoles, and the maximum horizontal dispersion is 1.74 meters. Sextupole and correction elements are located to one side of each arc quadrupole.

All six insertion regions have the same crossing geometry design, while their optics may be independently adjusted to tune the beam size at the collision points. The collision point beta function $\beta^{*}$ is nominally 10 meters for injection and acceleration, but can be reduced as low as 1 meter for high luminosity running. The standard collision point focusing technique uses a triplet of quadrupoles close to the collision point. Unfortunately this does not work at RHIC because of the need to collide ions of different species. Instead the beam paths, which are collinear at the collision point, are horizontally separated by special dipole magnets before they enter individual quadrupole triplets. While the free space available to the detectors is typical of other hadron colliders (about $\pm 9 \mathrm{~m}$ ), the presence of the separation dipoles pushes the insertion region triplets away from the collision point, and leads to $\beta$ values as large as 1450 meters. This still keeps about $5 \sigma$ of the beam within the "good field region" of the insertion region quadrupoles. 
The magnets are cooled to a temperature of $<4.6 \mathrm{~K}$ by circulating supercritical helium which is supplied by the existing $24.8 \mathrm{~kW}$ refrigerator. The beam tube in the superconducting magnets is at the temperature of liquid helium. An extremely good vacuum with a pressure < $10^{-11}$ Torr may be obtained in the absence of leaks into the cold bore. In order to avoid beam loss and radiation background, a vacuum of about $5 \times 10^{-10}$ Torr is required in the warm beam tube sections of the insertion regions.

\section{B. The Collider Scenario}

There are three major design goals for the collider. First, a specific energy of at least 100 $\mathrm{GeV} / \mathrm{u}$ (kinetic energy per nucleon) per beam is required. Second, an average luminosity of $2 \times 10^{26} \mathrm{~cm}^{-2} \mathrm{~s}^{-1}$ is required for the case where the two beams are gold ions. The luminosity is a measure of the density occupied in a given region by the particles in both beams as they cross at the collision point. The number of events per second corresponding to a specific reaction channel is given by the cross section for that channel in $\mathrm{cm}^{2}$ times the luminosity. Finally, the luminosity lifetime should be $\sim 10$ hours. That is, the luminosity is required to be high enough to be usable for that period of time during which the beams circulate and collide. The design luminosity as a function of both (selected) ion species and energy is shown iı Fig. III.2

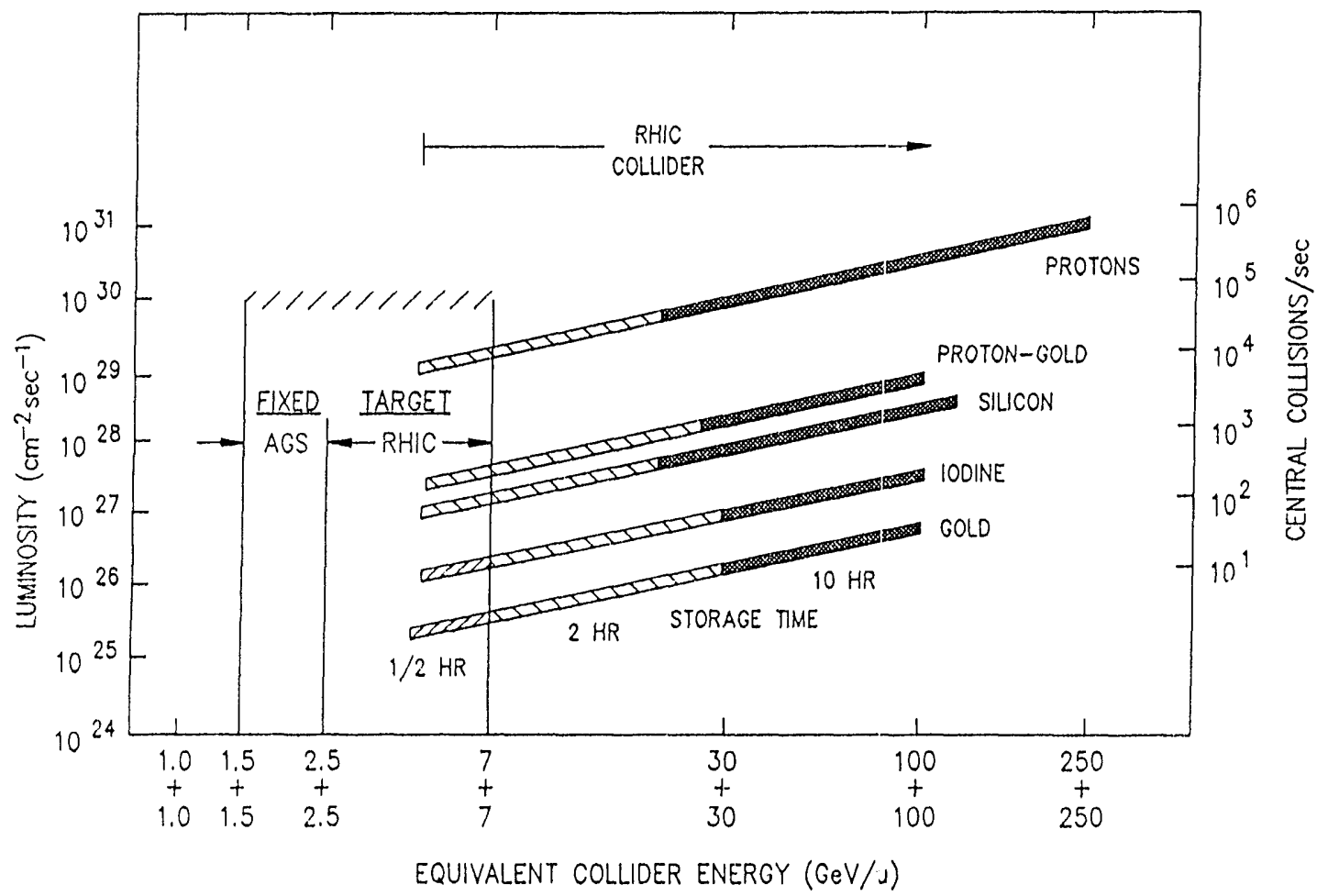

Fig. III.2 Design luminosity as a function of energy and species. Note that fixed target operation is also possible. 
The existing accelerator complex at BNL consisting of Tandem Van de Graaff accelerators, the Booster synchrotron, and the AGS, will serve as the injector for the RHIC. The sequence of steps in the chain of accelerators is shown in Fig. III.3 for the prototypical example of gold ions.

The existing upgraded Tandem Van de Graaff accelerators will serve for the initial ion acceleration. It is planned that a two-stage operation be employed with the negative ion source at ground potential. The negative ions, with charge -1 , are accelerated from ground to $+15 \mathrm{MV}$ potential. They pass through a stripping foil in the high voltage terminal yielding partially stripped

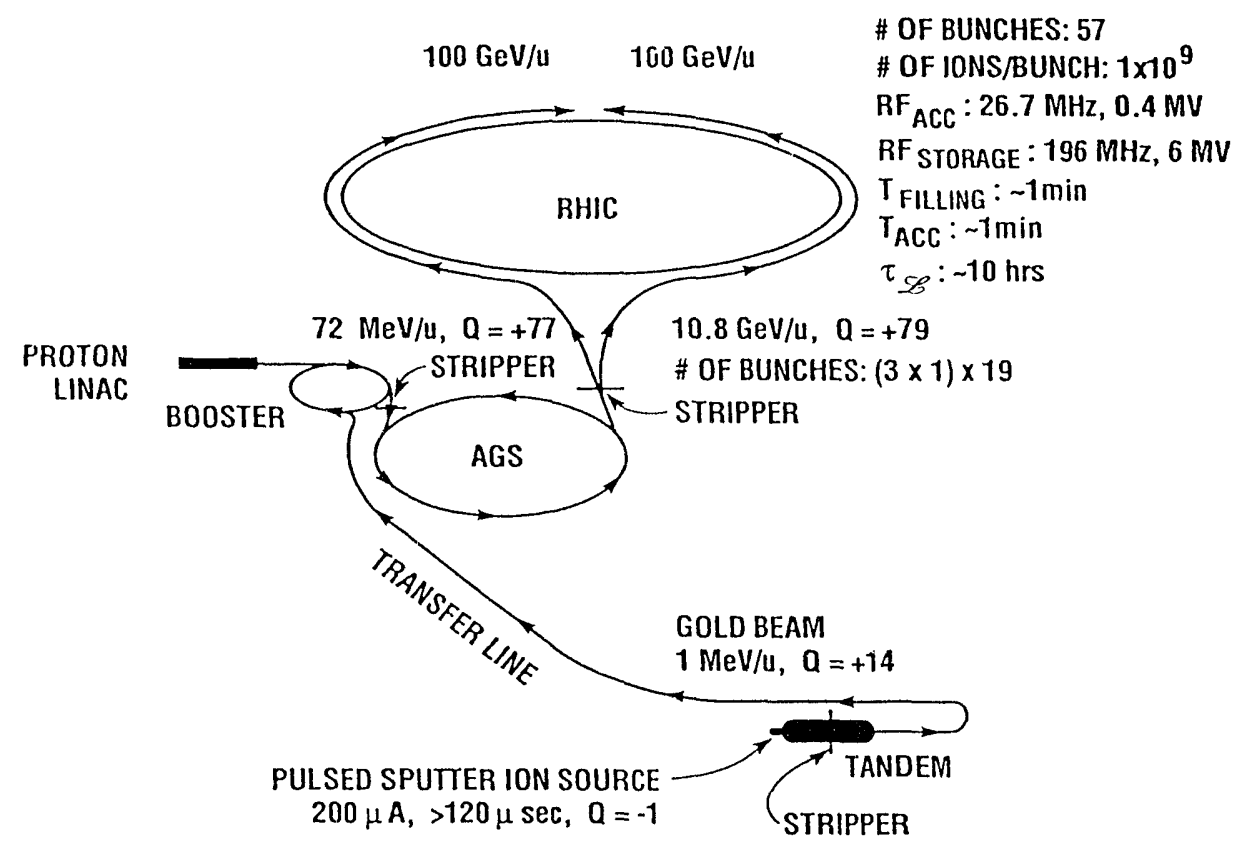

Fig. III.3 RHIC Acceleration Scenario for gold.

ions, with a positive charge QT, which is a function of the element being accelerated. The partially stripped ions are accelerated back to ground potential increasing their energy by $15 \times$ QT $\mathrm{MeV}$. For the prototypical example of gold beams, the ions exit the Tandem at the kinetic energy of $1 \mathrm{MeV} / \mathrm{u}$ and with $\mathrm{Q}=+14$ charge state.

Exiting from the Van de Graaff, the ions will traverse a long ( 550 m) heavy ion transfer line to be injected into the Booster synchrotron. The beam from the Tandem will be stacked in betatron (transverse) phase space by filling the machine with some number of consecutive turns. For gold, the total number of particles is expected to be $\sim 6 \times 10^{9}$. The beam is captured in the Booster using an of system operating at a convenient frequency. At the end of the Booster 
acceleration cycle, the particle bunch length will have contracted so that it will match the length of the rf buckets in the AGS. At a field of $1.27 \mathrm{~T}$, gold ions with $\mathrm{Q}=+14$ will have a kinetic energy of $72 \mathrm{MeV} / \mathrm{u}$ which corresponds to $\beta \gamma=0.4$

After extraction from the Booster, the ions pass through one more stripping target (about $70 \mathrm{mg} / \mathrm{cm}^{2}$ for gold ions), where they become stripped to a charge state of +77 and then enter the AGS. Each particle bunch train transferred from the Booster to the AGS is accelerated to the top AGS energy (28.3 GeV for protons; $10.8 \mathrm{GeV} / \mathrm{u}$ for gold corresponding to $\mathrm{B} \rho=100 \mathrm{Tm}$ ) and is then stripped of their last (K-shell) electrons and transferred to the collider by a magnet system installed in the existing transfer line tunnels. The transport system here will use conventional room temperature magnets, and will employ a magnetic septum and fast kicker system of wellproven design to deposit the beam vertically onto the injection closed orbit in the collider.

A total of nominal 57 bunches are injected into each ring in boxcar fashion. The AGS extraction system will allow single-bunch transfer of the three AGS bunches into one of the two collider rings. This cycle is repeated 19 times in order to fill one ring with the 57 bunches. Filling both rings requires about 1 minute. In the case of gold, there will be $\sim 1 \times 10^{9}$ ions/bunch or a total of $\sim\left(6 \times 10^{10}\right.$ ions in the 57 bunches in each ring. For the lightest ions, hydrogen and deuterium, approximately $1\left(0^{11}\right.$ ions/bunch can be stored in the collider as presently designed. The choice of 57 bunches is a direct consequence of the fact that the collider circumference is 4-3/4 times the AGS circumference and that the bunches are transferred equally spaced from the AGS to the collider. In the boxcar filling mode, the transfer is bunch-to-bucket and, therefore, the $\mathrm{rf}$ frequency must have an harmonic equal to an integer times 57 . The bunches are captured in stationary buckets of the so-called acceleration rf system operating at $\sim 26.7 \mathrm{MH} \%$, corresponding to a harmonic $h=57 \times 6$. The harmonic chosen and rf voltage of $215 \mathrm{kV}$ during beam transfer are well-matched to the AGS rf parameters. The acceleration rf system has 2 cavities per ring capable of providing a total of $400 \mathrm{kV}$ peak voltage.

The bunch separation is $67 \mathrm{~m}$ which corresponds to a rise time of $\sim 190 \mathrm{nsec}$ for the RHIC injection kicker: An upgrade option is to stack 114 bunches in RHIC which will require a kicker with $95 \mathrm{nsec}$ rise time. The injection kicker will allow the transfer of single bunches from the AGS, which will be the standard beam transfer mode.

Beam parameters at injection for an illustrative selection of ion species are given in Table III.1. It is assumed that, because of the required manipulations, the nominal Booster betatron emittance and the longitudinal phase space area are somewhat diluted to the final values shown in 
this table. The beam parameters are taken to be the same for all species except protons, which have a large emittance since they come from a different source. The normalized emittance containing $95 \%$ of the beam, $\varepsilon_{\mathrm{N}}$, is defined by $\varepsilon_{\mathrm{N}}=6 \pi \beta \gamma \sigma_{\mathrm{H}, \mathrm{V}}^{2} / \beta_{\mathrm{H}, \mathrm{V}}$ where $\sigma_{\mathrm{H}, \mathrm{V}}$ is the rms beam width or height and $\beta_{\mathrm{H}, \mathrm{V}}$ the horizontal or vertical amplitude lattice function. The bunch length given is the shortest one can obtain from the AGS. The nominal number of ions per bunch transferred to the collider is also given in Table III.1 It was derived after allowing for some losses in the Booster and the AGS. The beam parameters at the end of the acceleration cycle are also listed in Table III.I.

Table III.1 General Beam Parameters for the Collider

\begin{tabular}{|c|c|c|c|c|c|c|c|}
\hline Element & Proton & Deuterium & Oxygen & Silicon & Copper & Iodine & Gold \\
\hline Alomic Number $Z$ & 1 & 1 & 8 & 14 & 29 & 53 & 79 \\
\hline Mass Number $A$ & 1 & 2 & 16 & 28 & 63 & 127 & 197 \\
\hline \multicolumn{8}{|l|}{ Injection: } \\
\hline Energy, $\gamma$ & 31.2 & 15.6 & 15.7 & 15.7 & 14.5 & 13.1 & 12.6 \\
\hline Norm. Eniltance (xmm mrad) & 20 & 10 & 10 & 10 & 10 & 10 & 10 \\
\hline Bunch Length (III) & \pm 1.77 & \pm 2.33 & \pm 2.33 & \pm 2.33 & \pm 2.39 & \pm 2.43 & \pm 2.44 \\
\hline Energy Spreud $\left(\times 10^{-4}\right)$ & \pm 5.6 & \pm 8.4 & \pm 8.4 & \pm 8.4 & \pm 8.9 & \pm 9.7 & \pm 10.2 \\
\hline Nu iuns/Bunch $\left(\ldots 10^{1 /}\right)$ & 100 & 100 & 8.3 & 5.6 & 2.7 & 1.5 & 1.0 \\
\hline \multicolumn{8}{|l|}{ Tup Enerisy:" } \\
\hline Energy, ir & 268.2 & 134.2 & 135.2 & 135.3 & 124.5 & 112.9 & 108.4 \\
\hline Butich Length (m) & \pm 0.60 & \pm 0.84 & \pm 0.84 & \pm 0.84 & \pm 0.87 & \pm 0.92 & \pm 0.93 \\
\hline Energy Spread $\left(\times 10^{-1}\right)$ & \pm 1.92 & \pm 2.76 & \pm 2.75 & \pm 2.75 & \pm 2.82 & \pm 3.03 & \pm 3.05 \\
\hline \multicolumn{8}{|l|}{ Top Encrasy, 10 h: } \\
\hline rms Bunch Length (m) & 0.14 & & & & & & 0.25 \\
\hline Energy Spreinl $\left(\times 10^{-3}\right)$ & \pm 1.25 & & & & & & \pm 1.9 \\
\hline Norm. Emittance (xmm-mrad) & 30 & & & & & & 43 \\
\hline
\end{tabular}

Acceleration of System $h=3+2, V_{\mathrm{tl}}=300 \mathrm{kV}$.

'Sturage if System $h=2508, V_{\mathrm{r}}=0 \mathrm{MV}$

After having reached the operating energy, which takes about 1 min., the bunches are transferred from the acceleration $(26.7 \mathrm{MHz}$ ) to the storage of system at $196 \mathrm{MHz}$. The harmonic number of the storage of is $h=22 \times 114=2508$, resulting in a bucket length of $1.53 \mathrm{~m}$. This frequency was chosen in order to limit the growth of the bunch due to intrabeam scattering to an $\mathrm{rms}$ bunch length of $\sim 25 \mathrm{~cm}$. The resulting rms diamond length is a maximum of $\sim 18 \mathrm{~cm}$. The voltage requirement of the storage rf system is determined by the bucket half height, which has to accommodate the energy spread of the beam. In the case of Au bunches the initial voltage required is $\sim 770 \mathrm{kV}$. After transfer, the storage of voltage is adiabatically raised to the maximum value available (6 MV) and kept constant during the storage cycle. 
The stored energy is about $200 \mathrm{~kJ}$ per ring, small enough to be aborted onto an internal dump at the end of the storage period or in case of equipment malfunction. 'The beam will be dumped in a single turn $(13 \mu \mathrm{sec})$ by activating the ejection kicker which deflects the beam onto a dump block. In order to facilitate the beam abort design, a gap of $\sim 1 \mu \mathrm{sec}$ corresponding to 4 missing bunches will be provided.

Operation of the collider and achievement of full performance will require continuous monitoring of many beam characteristics, and appropriate beam instrumentation will be provided. A central control system will allow the control of, and communication among, the various collider systems. The major parameters of the collider are listed in Table III. 2 below.

Table III.2 Major Parameters for the Collider.

Kinetic Energy, Injection-Top (each beam), Au

protons

Luminosity, Au-Au@100 GeV/u \& 10 h av.

No. of bunches/ring

No. of Au-ions/bunch

Operational lifetimeAu@ $\gamma>30$

Diamond length

Circumference, $4-3 / 4 \mathrm{C}_{\text {AGs }}$

Number of crossing points

Free space at crossing point

Beta@ crossing, horizontal/vertical low-beta insertion

Betatron tune, horizontal/vertical

Magnetic Rigidity, $B \rho$ : @ injection

$$
\text { (a) top energy }
$$

No. of dipoles (192/ring +12 common)

No. of quadrupoles (276 arc +216 insertion)

Dipole field@100 GeV/u, Au

Arc dipole length, effective

Arc quadrupole gradient

Arc quadrupole length, effective

Cooldor n time, entire system

Filling time (each ring)

Beam stored energy

Acceleration time

$$
\begin{array}{rl}
10.8-100 & \mathrm{GeV} / \mathrm{u} \\
28.3-250 & \mathrm{GeV} \\
-2 \times 10^{26} & \mathrm{~cm}^{-2} \mathrm{sec}^{-1} \\
57 & \\
1 \times 10^{9} & \\
-10 & \mathrm{~h} \\
18 & \mathrm{~cm} \mathrm{rms} \\
3833.845 & \mathrm{~m} \\
6 & \\
\pm 9 & \mathrm{~m} \\
10 & \mathrm{~m} \\
2 & \mathrm{~m}
\end{array}
$$

$28.827 / 28.823$

$97.5 \mathrm{~T} \cdot \mathrm{m}$

$839.5 \mathrm{~T} \cdot \mathrm{m}$

396

492

$3.45 \mathrm{~T}$

$9.45 \mathrm{~m}$

71.2 $\mathrm{T} / \mathrm{m}$

$1.11 \mathrm{~m}$

$\sim 10$ days

$<1$ min

$-200 \mathrm{~kJ}$

$1 \mathrm{~min}$

Suggested Further Reading

H. Foelsche, et. al. "The Relativistic Heavy Ion Collider, RHIC," in "Advances of Accelerator Physics and Technology," H. Schopper, Ed., World Scientific Publishing. Co., Singapore, 1993, p. 104. 


\section{The STAR Detector}

The Solenoidal Tracker At RHIC (STAR) is designed to search for signatures of quarkgluon plasma formation and to investigate the behavior of strongly interacting matter at high energy density. The emphasis is on the measurement and correlation of global observables on an event-by-event basis and the use of hard scattering of partons to probe the properties of high density nuclear matter. The event-by-event measurement of global observables - such as temperature, flavor composition, collision geometry, reaction dynamics, and energy or entropy density fluctuations - is possible because of the very high charged particle densities, $\mathrm{dn}_{\mathrm{ch}} / \mathrm{d \eta} \approx$ 700 expected in nucleus-nucleus collisions at RHIC. Event-by-event fluctuations are expected in the vicinity of a phase transition, so this experiment will be sensitive to threshold behavior of the experimental observables as a function of energy density. Full azimuthal coverage with particle identification and continuous tracking is required to perform these measurements at momenta where the particle yields are maximal.

To meet the design criteria and fulfill the physics objectives, tracking, momentum analysis and particle identification of most of the charged particles at midrapidity are necessary. The tracking must operate in conditions at higher than the expected maximum charged particle multiplicities ( $n_{\mathrm{ch}}=3000$ in the $|\eta|<1$ at midrapidity within the acceptance of the experiment) for central Au $+\mathrm{Au}$ collisions. The detection system (Fig. IV.1) will consist of a time projection chamber (TPC) and silicon vertex tracker (SVT) located inside a solenoidal magnet with $0.5 \mathrm{~T}$ field to provide tracking, momentum analysis and particle identification of most of the charged particles at midrapidity. Momentum measurements will be made over a large pseudo-rapidity range $(|\eta|<2)$ with full azimuthal coverage $(\Delta \phi=2 \pi)$. Particle identification via ionization energy loss $(\mathrm{dE} / \mathrm{dx})$ of pions/kaons for $\mathrm{p}<0.7 \mathrm{GeV} / \mathrm{c}$ and kaons/protons for $\mathrm{p}<1 \mathrm{GeV} / \mathrm{c}$ will be performed within $|\eta|<1$. In addition, measurement of decay particles and reconstruction of secondary vertices will be possible. A two-track resolution of $2 \mathrm{~cm}$ at $2 \mathrm{~m}$ radial distance from the interaction is expected. Momentum resolution of $\Delta \mathrm{p} / \mathrm{p} \approx 0.02$ at $\mathrm{p}=0.1 \mathrm{GeV} / \mathrm{c}$ is required to accomplish the physics, and $\Delta \mathrm{p} / \mathrm{p}$ of a few percent at $\mathrm{p}=10 \mathrm{GeV} / \mathrm{c}$ is sufficient to accurately measure the rapidly falling spectra at high $\mathrm{p}_{t}$ and particles from mini-jets and jets.

The TPC will be divided into two longitudinal drift regions, each 2.1 meters in length (Fig. IV.2). The two drift regions are separated by a high voltage membrane at $\eta=0$. Electrons created from track ionization drift in the longitudinal direction along the TPC electric field lines to the end caps of the TPC. Each end cap is instrumented with 70,000 pads, and each pad read out in 512 


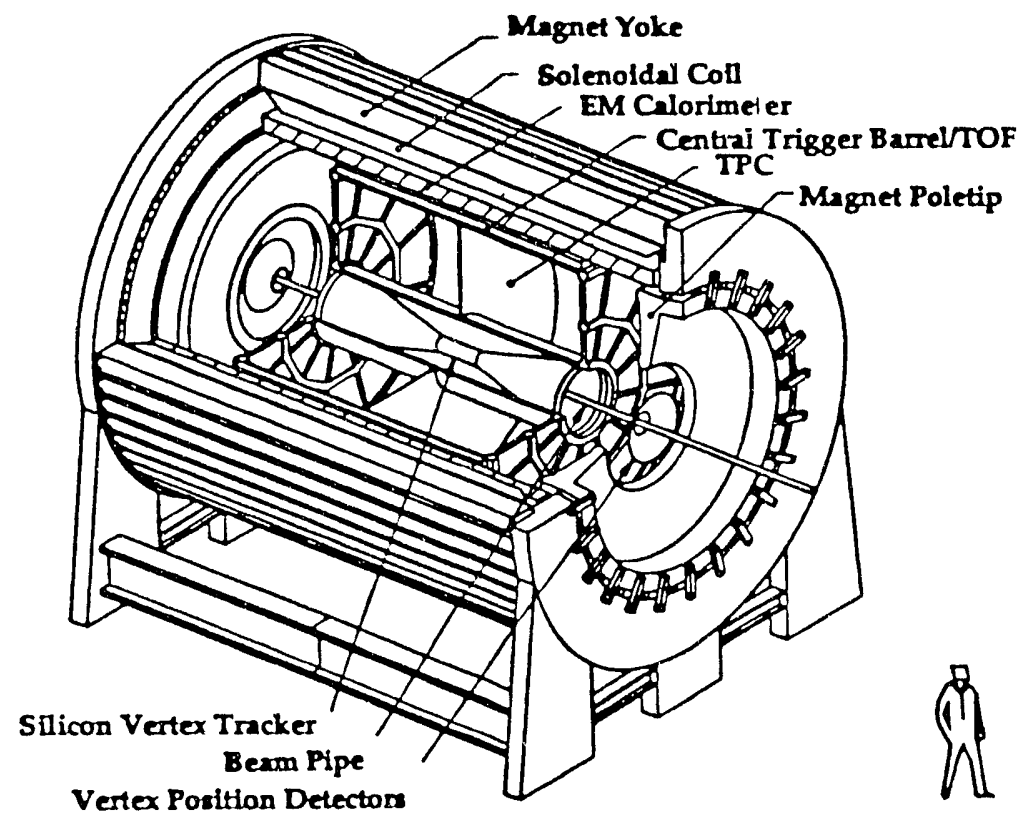

Fig. IV.1 A perspective view of the STAR experimental configuration

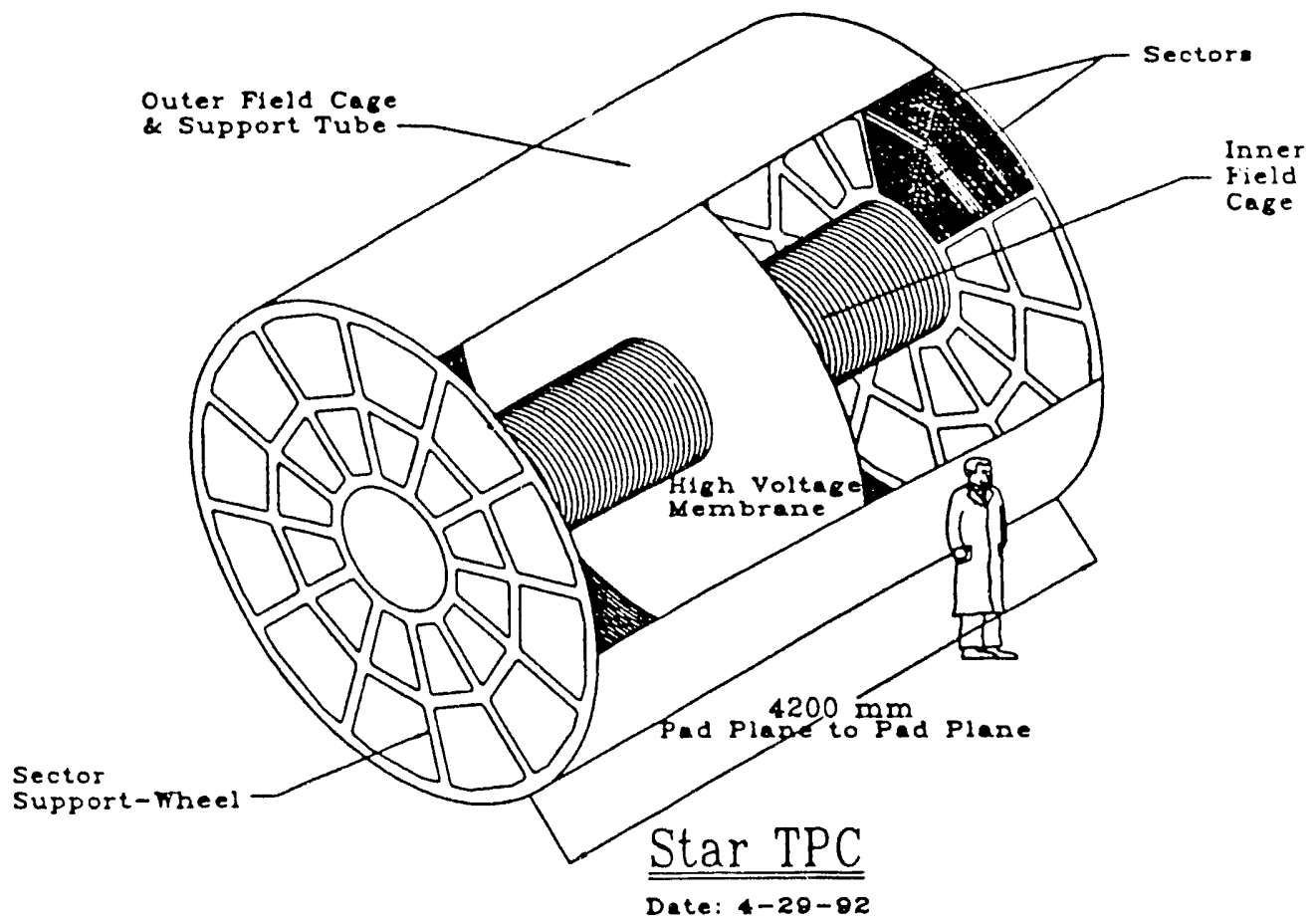

Fig. IV.2 A perspective view of the STAR Time Projection Chamber

time samples during the $50 \mu$ see required to drift the ionization from the midplane to the padplane of the TPC volume. The inner radius of the TPC will be $50 \mathrm{~cm}$. and the outer radius 2()() $\mathrm{cm}$. Neglecting energy loss, singly ionized particles having $\mathrm{Pt} \leq 40 \mathrm{MeV} / \mathrm{c}$ spiral inside the TPC inner radius, and do not reach the active volume of the TPC. For these particles, the silicon vertex 
tracker will be used as a standalone device. Particles having $\mathrm{p}_{\mathrm{t}} \geq 150 \mathrm{MeV} / \mathrm{c}$ traverse the TPC, and exit at the outer radius. Given the simulated response of the TPC and the high charged particle densities expected in nucleus-nucleus collisions at RHIC, it will be possible, for example, to accurately determine the slope of the pion $p_{t}$ distribution on an event by event basis (Fig. IV.3), and to search for critical dynamical fluctuations in a narrow range of conditions as a possible indicator of a transition to a deconfined phase of quarks and gluons.

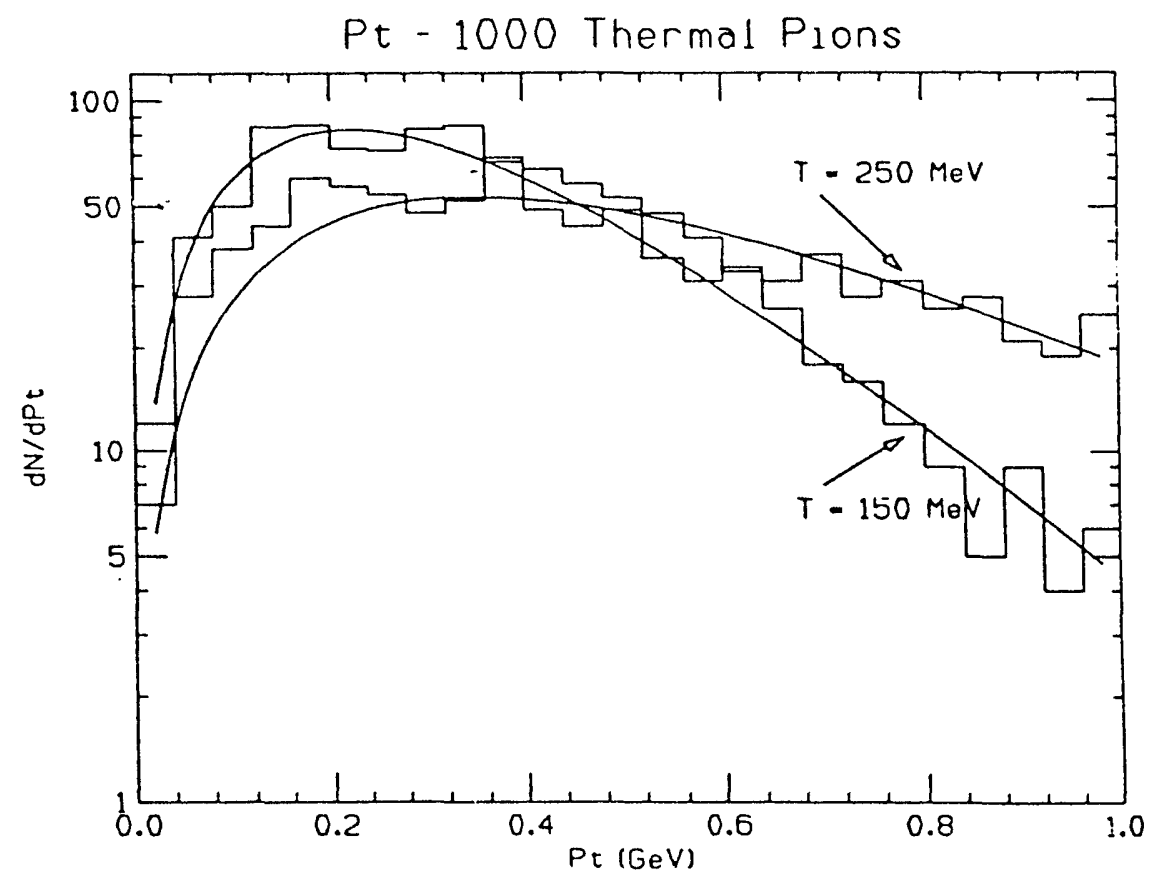

Fig. IV.3 Simulation of the $p_{t}$ spectrum for one event generated using a Boitzmann distribution of 1000 pions. The histrograms correspond to single events generated with $\mathrm{T}=150 \mathrm{MeV}$ and $250 \mathrm{MeV}$. The curves are fit to the histograms using a Boltzmann distribution.

The silicon vertex tracker (SVT) coupled with the TPC will locate the position of the primary vertex to high accuracy, improve the momentum and $\mathrm{dE} / \mathrm{dx}$ resolutions, and locate secondary vertices to an accuracy of better than $100 \mu \mathrm{m}$. It presently consists of 168 silicon drift detectors arranged in three concentric barrels around the interaction point at radii of 5,8 , and 13 $\mathrm{cm}$ respectively. It is designed to provide three dimensional space points for track segments within its acceptance, and therefore will provide a powerful tool for extending the acceptance of the TPC to low momenta. The improved vertex resolution of the SVT will also make it possible to measure the decay of baryons and anti-baryons having multiple strangeness (e.g. $\Xi^{--}$, cf. Fig. IV.4), whose yield is sensitive to the strangeness density reached in nucleus-nucleus collisions, and is therefore a possible signature of quark gluon plasma formation. 


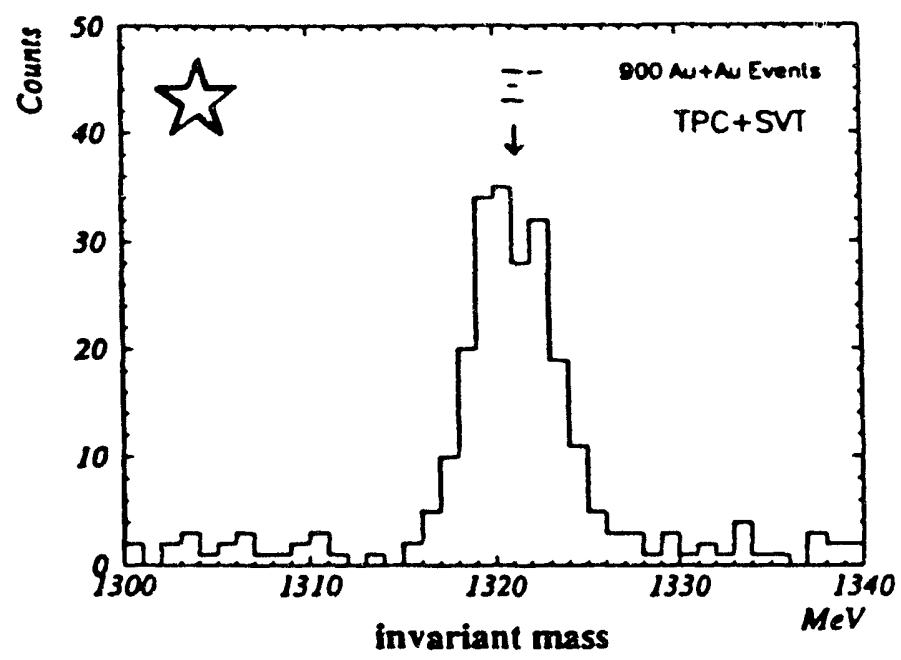

Fig. IV.4 Reconstructed invariant mass distribution of the $\Xi^{-}(\mathrm{TPC}+\mathrm{SVT})$ with TPC momentum resolution taken into account.

A central trigger bariel around the TPC, and veto calorimeters located in the region of the beam insertion magnets will provide the information necessary for a collision geometry trigger by measuring the multiplicity at midrapidity and the energy carried forward by spectator nucleons, respectively. Vertex position detectors near the beamline at either end of the solenoidal magnet have been designed to localize the interaction to within $\pm 6 \mathrm{~cm}$ in $\leq 100 \mathrm{~ns}$. Precise determination of the vertex position will be accomplished using the TPC and SVT detectors. A portion of the central trigger barrel will be instrumented with a highly segmented time of flight (TOF) array. Each TOF scintillator will be approximately $1.5 \mathrm{~cm} \times 2.6 \mathrm{~cm} \times 23.5 \mathrm{~cm}$ in dimension. and will provide TOF resolution on the order of 100 picoseronds. For particles within the acceptance of the TOF array, the pion/kaon separation will be extended from $\sim .7 \mathrm{GeV} / \mathrm{c}$ to $\sim 1.3 \mathrm{GeV} / \mathrm{c}$, and the kaon/proton separation will be extended from $\sim 1 \mathrm{GeV} / \mathrm{cto} \sim 2.4 \mathrm{GeV} / \mathrm{c}$.

An electromagnetic calorimeter (EMC) located outside the central trigger barrel will be used to trigger on local ( $\left.\mathrm{d}^{2} \mathrm{E}_{\mathrm{l}} / \mathrm{d} \eta \mathrm{d} \phi\right)$ and global ( $\mathrm{E}_{\mathrm{l}}$ ) transverse energy, and to measure jets, direct photons. and leading $\pi^{\circ}$ production. Correlation of the information from the EMC and central trigger barrel detector systems will also enable STAR to trigger on events exhibiting unusual pion isospin abundances (Centauro events) which have been suggested as a signature of exotic nuclear matter in nucleus-nucleus collisions at high energy. The STAR EMC is a sampling calorimeter $21 X_{0}$ thick. constructed of alternating layers of lead absorber and scintillator tiles read out with wave shifting fibers. It is located interior to the magnet coil. and is designed to have a resolution of $20 \% / \mathrm{V}$. Full calorimetry in STAR will be accomplished using a combination of TPC tracking and electromagnetic calorimetry 
External TPC's located at \pm 7 meters from the interaction point along the beamline will be used to study the transfer of energy from the projectile rapidity to midrapidity by following the fate of the incident baryons rescattered in the collision. These chambers extend the pseudorapidity coverage of STAR from $|\eta|=2$ to $|\eta| \leq 4$. By analyzing the difference in the distribution of positively and negatively charged particles in this acceptance, nuclear stopping and possible collective effects in spectator matter will be investigated. Extension of the tracking acceptance to I ! $\leq 4$ should enhance the ability of STAR to resolve events and regions of anomalously high particle production which could result from the hadronization of a quark gluon plasma.

\section{Suggested Further Reading}

"Conceptual Design Repont for the Solenoidal Tracker at RHIC." The STAR Collaboration, PUB-5.547, LBL. June 15. 1992 . 


\section{The PHENIX Detector}

The primary goals of the PHENIX experiment are to detect a new phase of matter, the quark-gluon plasma (QGP), and to measure its properties. Many of the potential signatures for the QGP are measured as a function of a well-defined common variable related to the energy density to see if any or all of these signatures show a simultaneous anomaly due to the formation of the QGP. To achieve these goals the strategy of PHENIX is to study leptonic, photonic and hadronic signatures in the same experiment. In addition, basic quantum chromodynamics phenomena, collision dynamics, and thermodynamic features of the initial states of the collision are studied.

The PHENIX collaboration searches for the QGP through a programmatic study of a broad array of potential signatures as a function of energy density in both $A+A$ and $p+A$ collisions. Lepton pairs (dielectrons and dimuons) are measured to study various properties of vector mesons, such as the mass, the width, and the degree of yield suppression due to the formation of the QGP. The effect of thermal radiation on the continuum is studied in different regions of rapidity and mass. The $e \mu$ coincidence is measured to study charm production, and to help understand the shape of the continuum dilepton spectrum. Photons are measured to study direct emission of single photons and to study $\pi^{\circ}$ and $\eta$ production. Charged hadrons are identified to study the spectrum shape, production of antinuclei, the $\phi$ meson (via $\mathrm{K}^{+} \mathrm{K}^{-}$decay), jets, and two-boson correlations. Since the special emphasis of PHENIX is to probe the properties of matter from simultaneous measurements of all these particles, particle identification is an important aspect of this experiment. The measurements are made down to small cross sections to allow the study of high $p_{\mathrm{T}}$ (transverse momentum) spectra, and $\mathrm{J} / \psi$ and $\Upsilon$ production. Recognizing that some of the potential QGP signatures involve rare processes and small effects, PHENIX is designed to be a detector capable of taking data at the highest luminosities expected at RHIC.

The PHENIX detector is shown schematically in Fig. V.1. There are two large magnets, labeled the central magnet $(\mathrm{CM})$ and the muon magnet $(\mathrm{MM})$. The height of the $\mathrm{CM}$ is approximately $9.5 \mathrm{~m}$; it weighs nearly 500 tons; and it produces an azimuthally symmetric magnetic field (with principal component parallel to the beam directions) with a field strength of up to $9 \mathrm{kG}$ at $r=0$. The height of the $\mathrm{MM}$ is approximately $10.5 \mathrm{~m}$; it weighs more than 400 tons; and it produces a radial magnetic field with a field integral along $\theta=15^{\circ}$ of $0.72 \mathrm{~T} \cdot \mathrm{m}$. Fig. V.1 also shows the three sets of PHENIX detector subsystems: the inner detectors, which cover 2- 
$178^{\circ}$ in polar angle $\theta$ and $0-360^{\circ}$ in azimuthal angle $\phi$, the central arms, which cover $70-100^{\circ}$ in $\theta$ and $\pm\left(22.5-112.5^{\circ}\right)$ in $\phi$, and the muon arm detectors, which cover $10-35^{\circ}$ in $\theta$ and $0-360^{\circ}$ in $\phi$.

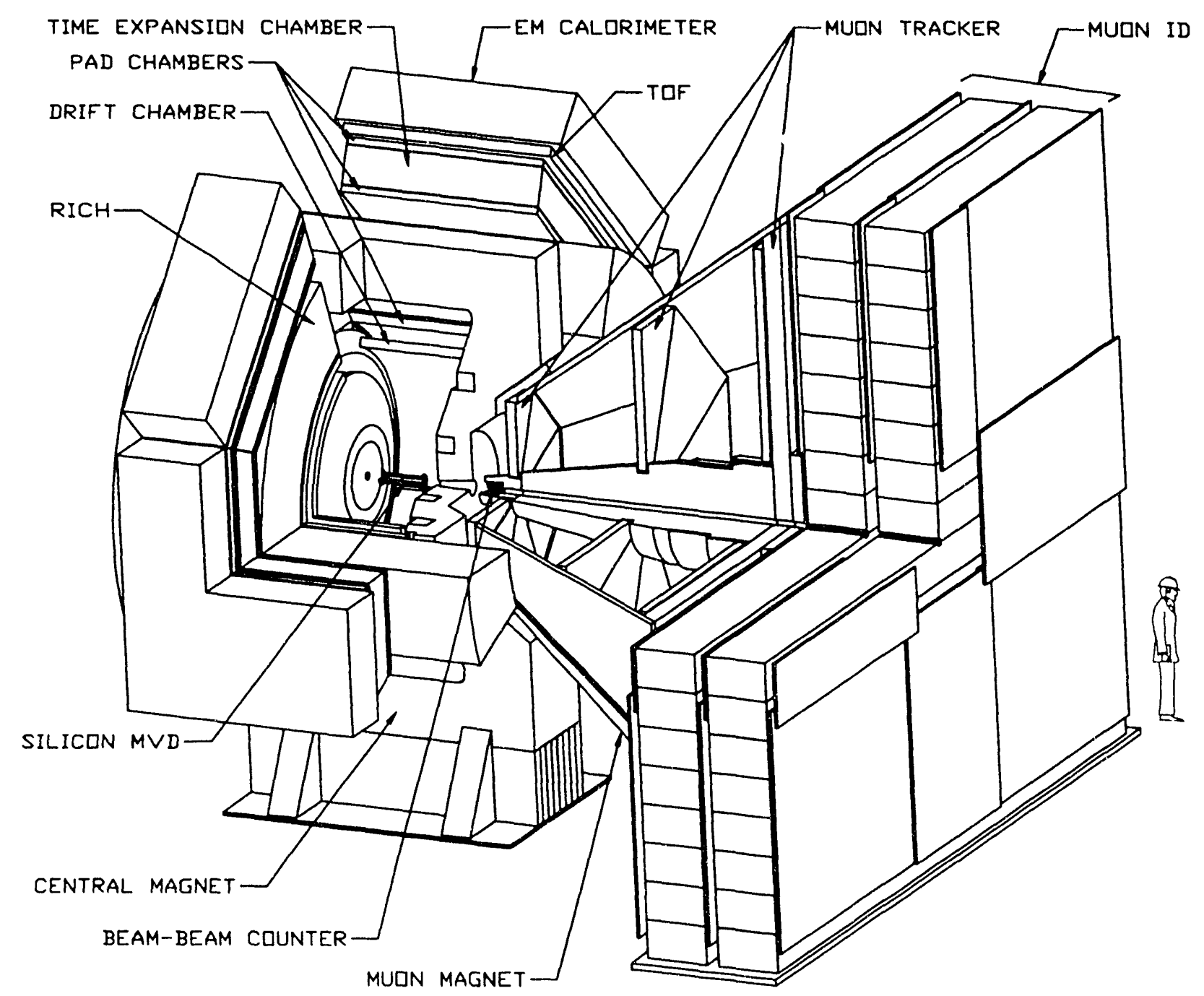

Fig. V.1 A three-dimensional cutaway view of the PHENIX detector, showing the location of the various detector subsystems. The two large magnets and the PHENIX detector subsystems are labeled in the figure.

The inner detectors are those closest to the interaction point. The two beam-beam (BB) counters. located upstream and downstream of the interaction point, are arrays of Cherenkov counters whose purpose is to provide a rough $(\sigma, \sim 2 \mathrm{~cm})$ vertex location and a precise time $\left(\sigma_{t}<\right.$ 100) $\mathrm{ps}$ ) for the event. The silicon MVD (multiplicity vertex detector), which has over 43,00() channels, will enable detailed measurements of charged particle multiplicity and a precise $(\sigma,-$ $50(0) \mathrm{m})$ vertex location. 
The central arms of PHENIX comprise many detectors used for both particle identification and tracking. The Drift Chamber provides precise $(\sim 150 \mu \mathrm{m})$ measurements of charged particles in the $r-\phi$ direction to determine the transverse momentum of particles and, ultimately, the invariant mass of particle pairs. The RICH (Ring Imaging Cherenkov Detector) allows electron identification up to $\sim 4 \mathrm{GeV} / \mathrm{c}$. The PAD Chambers provide non-projective tracking information and make the principal $z$-coordinate determinations. The TEC identifies electrons up to $\sim 2 \mathrm{GeV} / \mathrm{c}$ by $d \mathrm{E} / d x$ measurement. The TEC also identifies $\pi, \mathrm{K}$, and p over a limited energy range. The TOF (time-of-flight) detectors, with timing resolution $<80$ ps, provide hadron identification and are able to separate $\pi$ and $\mathrm{K}$ at the $5 \sigma$ level up to $2.4 \mathrm{GeV} / \mathrm{c}$. Finally, the EM (Electromagnetic) Calorimeter, when given the charged track location and momentum information from other detectors, allows photon identification and reconstruction, as well as electron identification from energy and time measurements.

The muon arm of PHENIX comprises the MM magnet, the Muon Tracker, and the Muon ID (identifier). Its main purpose is to detect muon pairs originating from vector meson decay or virtual photon production. The Muon Tracker comprises three sets of multi-plane drift chambers, which measure the charge and momenta of particles. The Muon ID is several layers of steel absorber (instead of the concrete layers indicated in Fig. V.1) interleaved with limited streamer tube detectors.

The electronics for the PHENIX experiment and the On-Line System are designed to handle signals coming from the thirteen specific sets of detectors, to apply selective triggers to the data stream, and to record data on tape at a very high rate (in excess of $20 \mathrm{MB} / \mathrm{s}$ ). The front-end electronics are custom monolithic circuits tailored to the specific detectors. The trigger system consists of three levels, each pipelined and event-driven, achieving deadtimeless operation up until ADC conversion. Events can be built at a data rate of up to $1 \mathrm{~GB} / \mathrm{s}$, with high-level triggers and routing applied to full events. The recording rate requirement is very important, because of the large event size $(200 \mathrm{kB})$ and high luminosities expected at RHIC. For example, the particle multiplicity per event can exceed 10,000 for central $\mathrm{Au}+\mathrm{Au}$ events.

To quantitatively assess the performance of the entire PHENIX detector for each potential signature of the QGP, an integrated simulation computer package called PISA (PHENIX Integrated Simulation Application) was developed. As representative examples of the results from PISA, Fig. V.2 shows the simulated spectra for the entire invariant mass continuum expected for dielectrons (central arms) and dimuons (muon arm). Note that the $\omega, \phi, J / \psi$, and $\Upsilon$ 
vector mesons are clearly observable above the expected combinatoric backgrounds. The PISA simulations not only establish the feasibility of measuring the vector mesons and other particles produced in the collisions at RHIC, but also ensure that all of the PHENIX detector subsystems are optimally designed to achieve the physics goals of the PHENIX collaboration.
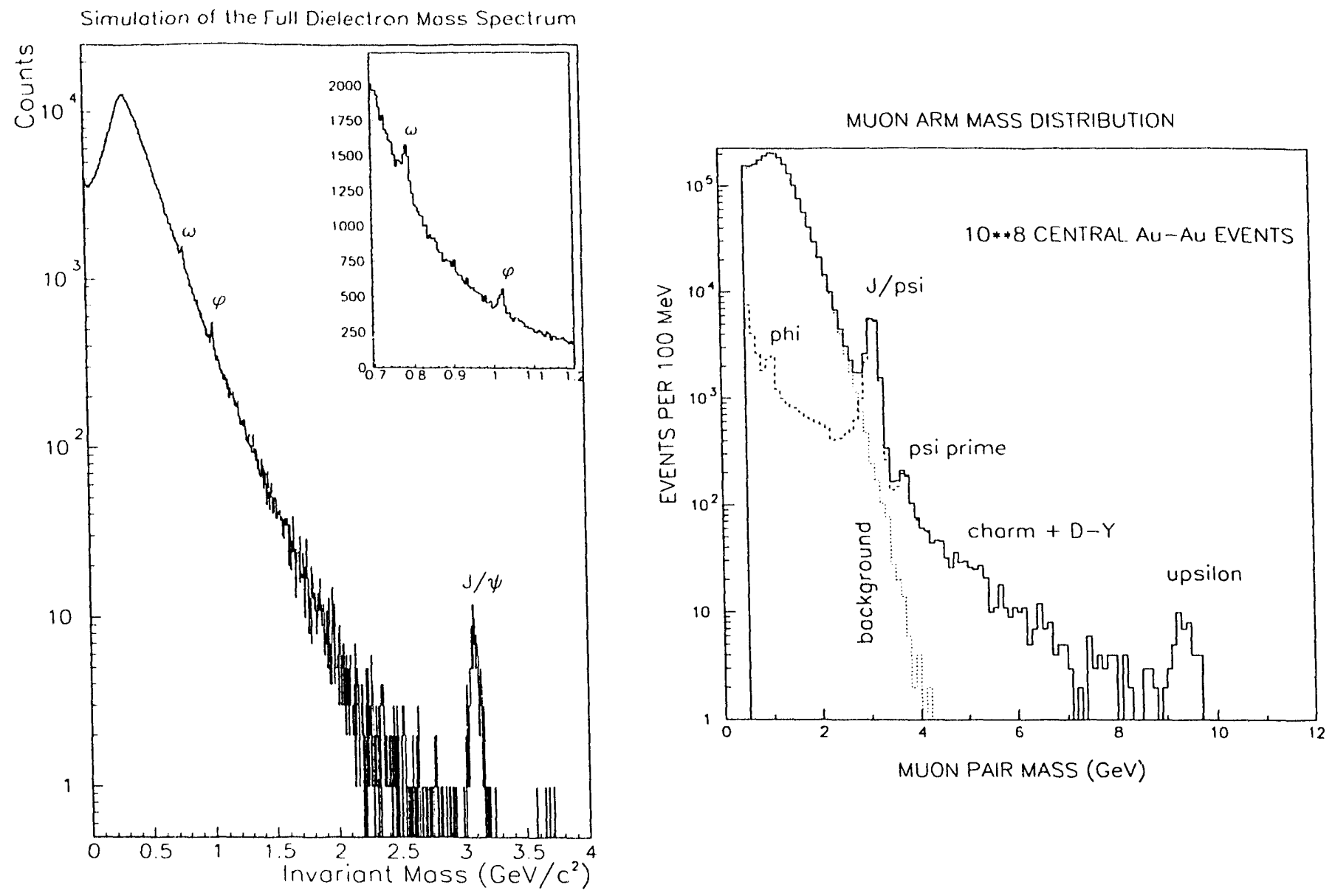

Fig. V.2 The full dielectron and dimuon pair mass regions, showing the $\omega, \phi, J / \psi$, and $\Upsilon$ vector mesons at their expected rates.

The PHENIX collaboration consists of over 300 scientists, engineers, and graduate students from 43 institutions in 10 countries. This large international collaboration is supported by US resources and significant foreign resources.

\section{Suggested Further Reading}

PHENIX Conceptual Design Report, 29 January 1993. 


\section{The PHOBOS Detector}

When RHIC becomes operational, gold nuclei will be made to collide head-on at unprecedented energies. All indications are that at RHIC some, if not all, head on gold-gold collisions will yield an energy density sufficiently high to produce the quark-gluon plasma (QGP). It is a new phase of matter that probably does not at present exist anywhere in the Universe, but which was the state of the whole Universe at about a millionth of a second after the big bang. The discovery and study of the properties of the QGP are the prime goals of the RHIC research program.

If a QGP is formed there are other exciting prospects for RHIC. For example, after formation the QGP will expand and cool. Once the temperature (and energy density) drops below a critical value hadronic particles will begin to form through coalescence of the various quarks, antiquarks and gluons. In a head-on gold-gold collision at RHIC, according to present predictions, as many as 15,000$)$ particles may be produced. Most of these particles are produced at the expanding boundary of the QGP. It is highly likely that in some collisions the produced particles will form a completely closed expanding shell. Should that occur a vacuum will appear in the middle of the shell. This vacuum will be particularly interesting since the ordinary vacuum is not unique. There are always fluctuations in the vacuum and the fluctuations are correlated. If two vacua have never been in contact they could both be in the lowest energy state but with different corielations in the fluctuations. We say that the two vacua have different spontaneously broken symmetry states. This is analogous to different possible orientations of the magnetization of a ferro-magnet below the Curie temperature.

If the vacuum inside this expanding shell of particles is different from the vacuum outside, something exciting should happen when the shell breaks. The two vacua make contact, and the little one has to re-align in the direction of the big one. During re-alignment, the surface energy is released in the form of many low energy-charge correlated pions. The detection of different states of the vacuum would be exciting indeed, and is just one example of the unexpected phenomena that might occur once the unprecedented energy and matter densities are actually produced and studied.

One of the research programs planned for the study of RHIC physics is PHOBOS. The PHOBOS detector consists of two almost - independent parts. The first is an array of counters with which, for each collision, it is possible to measure the number and direction of almost all the produced particles. The second is a high precision instrument with which the detailed properties of all the particles produced into two narrow cones, covering about $1 \%$ of the total solid angle, are measured.

The decision in PHOBOS to focus on these particular measurements is based on the following very general considerations: 
In relativistic collisions the angle at which a particle is produced is highly correlated with the velocity of the source from which the particle originated. For example, particles originating from a fast moving source will be produced at small angles relative to the incident beams, whilst particles originating from a source at rest in the center of mass system of the colliding nuclei will be produced at large angles.

Not knowing where the QGP might be produced, PHOBOS was designed to study the particles going in all possible directions. Hence the array of counters detects almost all the produced particles. Not knowing how often new physics might be produced, it is important to study as many gold-gold collisions as possible. For this to be economically and practically possible it is necessary to reduce the measurements on each produced particle to a minimum. Hence it was decided to measure, for most particles, only the production angle.

One of the characteristic features of phase transitions is fluctuations in physical observables. Such fluctuations can occur on any scale. In PHOBOS the arrays detecting all the particles have a fine granularity. They are thus ideally suited to study both the fluctuations of the overall number of produced particles from collision to collision and the local fluctuations in the individual collisions. Furthermore, the counters in the arrays are designed to differentiate between produced photons and charged particles. This will allow us to study the fluctuations in the ratio of charged to neutral pions that might occur, as discussed above, if different vacua are formed.

Once possibly interesting collisions are identified through the study of the overall characteristics of all the produced particles, it will be necessary to investigate in greater detail some of the collision products. Hence there will be within PHOBOS a high precision instrument, a two arm magnetic multiparticle spectrometer, with which the momenta of $1 \%$ of the produced particles will be measured and the particle species identified. With these spectrometers it will be possible to study the momentum spectra down to very low values, as well as particle ratios and correlations between particles. These quantities, in turn, will give information on the size of the interaction volume and the mass and width of the $\phi$-meson. PHOBOS concentrates on these particular measurements for the following reasons.

As discussed earlier, one indication that a QGP might have been formed is a source of produced hadrons whose dimensions are much bigger than the incident nuclei. Bose-Einstein correlations give a direct measurement of the source size. A copious production of particles of very low momentum, according to the uncertainly principle would be another indication of a large production volume. The transverse momentum of particles is related to the temperature of the source, while the total number of particles produced is related to the energy density. Observation of a discontinuity in the average transverse momentum at some value of the multiplicity of the produced particles would be a clear indication of the existence of two phases. Finally, ratios of particles with different quark compositions, and the masses and lifetimes (widths) of highly unstable particles such as the $\phi$-meson, are sensitive probes of the medium in which they are formed or in which they decay. Significant differences from the observations in proton-proton collisions could be indications of the production of a new kind of medium. 
Fig. VI.l illustrates the essential features of the PHOBOS detector. It also gives a summary of its properties. A preliminary engineering drawing of significant parts of the PHOBOS detector is shown in Fig. VI.2. The array of counters for detecting most of the particles (the multiplicity detector) consists of silicon strip detectors surrounding the interaction region and a set of circular silicon pad detectors located down- and up-stream from the interaction point at distances of up to 6 meters. Lead radiators placed in front of alternate pads are used to detect gammas.

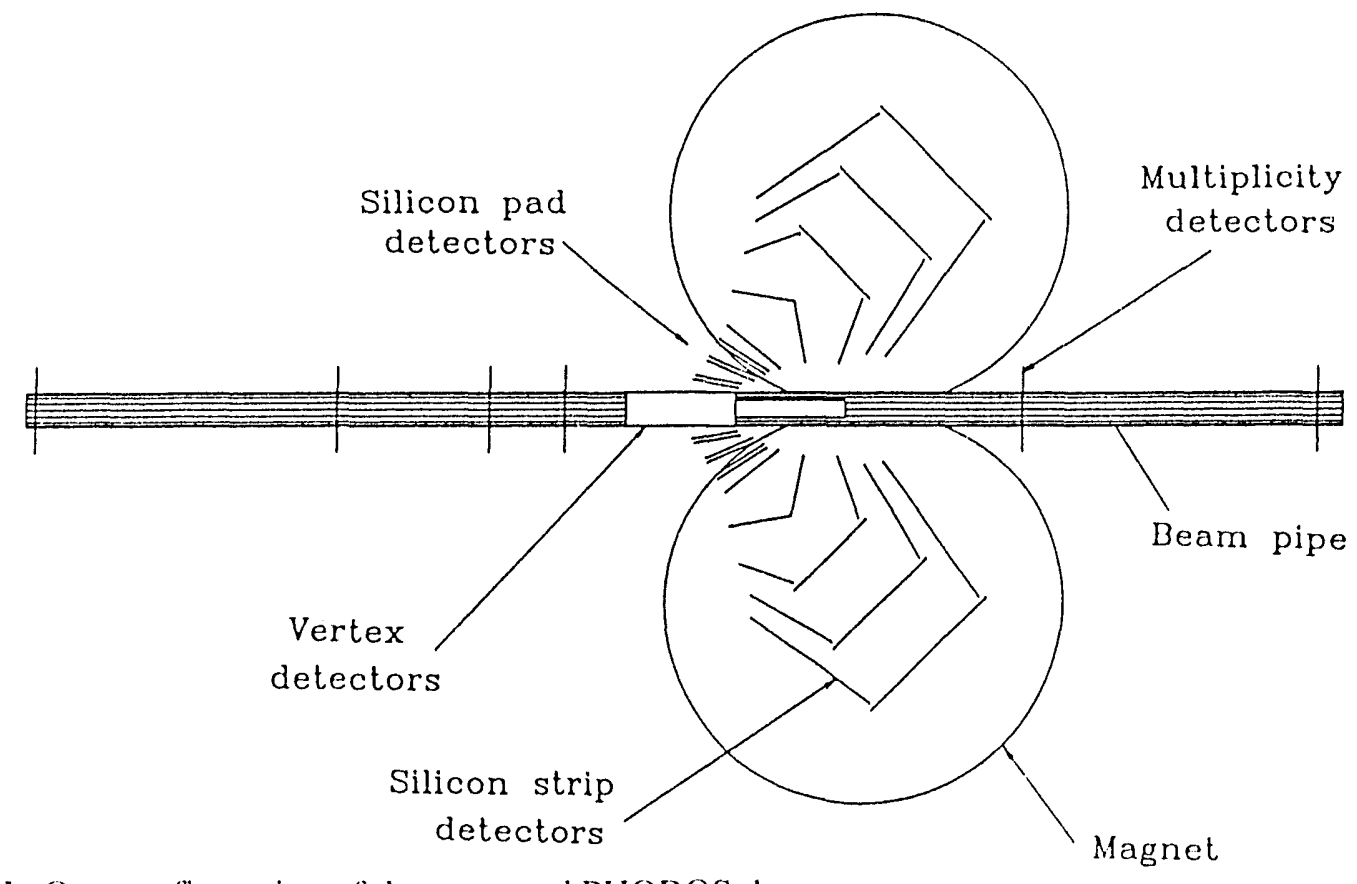

Fig. VI.I. One configuration of the proposed PHOBOS detector.

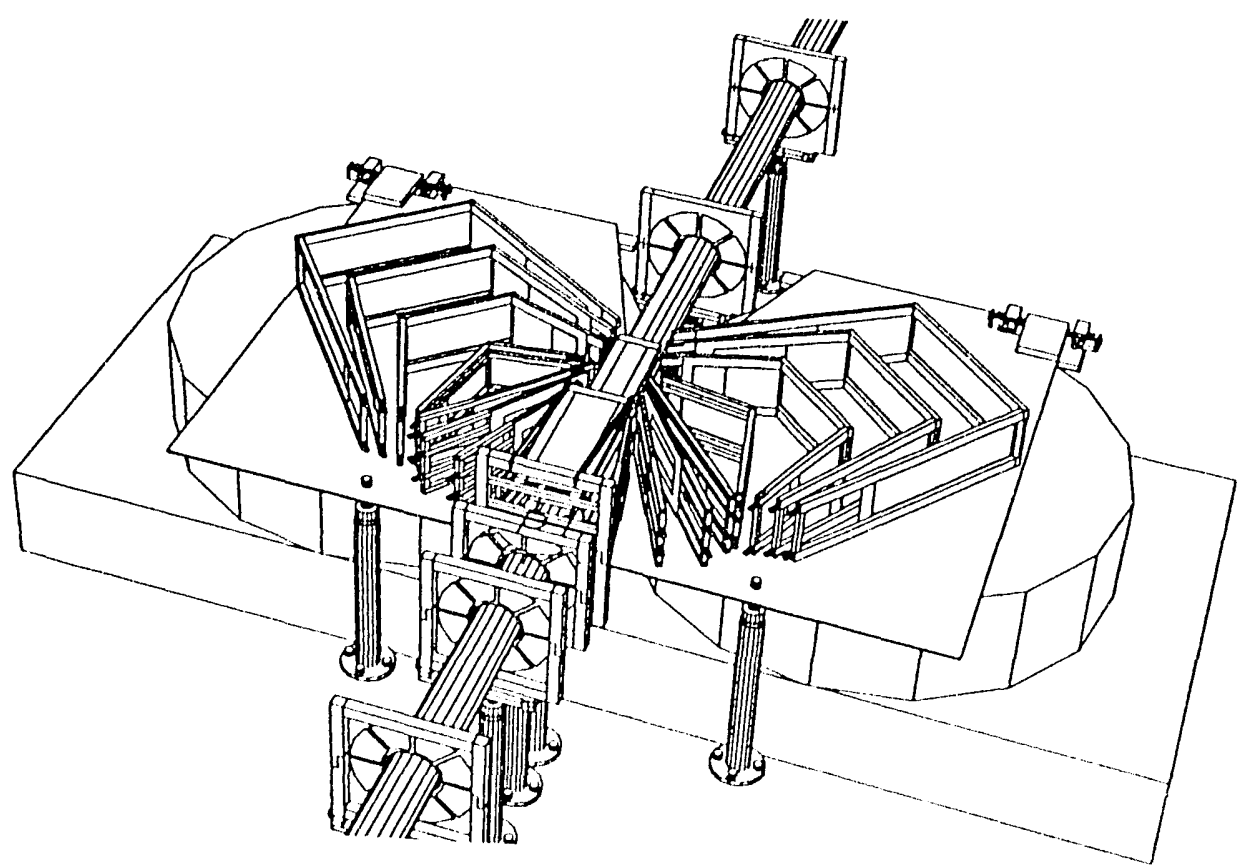

Fig. VI.2 A Preliminary engineering of significant parts of the PHOBOS detector. The top coils and flux return steel are not shown for clarity. 
The two arms of the multiparticle spectrometer are installed inside conventional magnets with a maximum field strength of 2 Tesla. The useful magnetic field occupies two cylindrical volumes; each of them is about $60 \mathrm{~cm}$ in diameter and $14 \mathrm{~cm}$ high. They are located on either side of the interaction volume and displaced with respect to the beam intersection point so that particles with transverse momenta down to $15 \mathrm{MeV} / \mathrm{c}$ can enter the magnetic volumes. The particle tracks in the magnetic field are recorded by 11 layers of silicon detectors. The first 5 layers are equipped with silicon pad detectors; they are located in the region of weak field and provide the angle measurement for particles emerging from the collision. The remaining 6 layers are equipped with strip detectors; they are positioned in a region of high field and provide momentum measurement. There are in total about 60,000 elements in the silicon detectors. The very large number of elements is necessary if we are to reconstruct particles that are close together. It is also necessary for the measurement of the momenta of particles with a precision adequate, for example, for the determination of the mass and width of the $\phi$-meson.

An important feature of the PHOBOS silicon detectors is that the pulse height information is recorded for every channel. In the multiplicity detector the pulse height is used to count the number of particles traversing each element. In the spectrometer this information is used to identify low momentum pions, kaons and protons. To further identify particles a time of flight hodoscope is placed at the back of the spectrometers.

With its wealth of complex instrumentation and powerful analytic capabilities, the PHOBOS detector, using the very newest technology and the very latest physics, will open a window on the very earliest universe. 


\section{The BRAHMS Detector}

At RHIC opportunities exist for experiments investigating both the quark gluon plasma at high temperature and low baryon density in the midrapidity region, and the baryon rich plasma in the fragmentation regions of rapidity. The BRAHMS detector, whose name is an acronym for Broad RAnge Hadron Measuring Spectrometers, is designed to explore a rapidity interval large enough to encompass both of the kinematic regions where transition to the plasma state may be expected to occur.

The most basic information available for understanding the phenomena that occur in heavy ion collisions comes from the momentum spectra and yields of the various emitted particles as a function of transverse momentum, $p_{t}$ and rapidity, $y$. It is one of the goals of this experiment to measure these spectra in the kinematic regions in which they are most sensitive to model predictions. Particle yields, as a function of $y$, are important indicators of the densities obtained in the collisions and of the produced entropy. The spectral shapes and slopes and their $y$ dependence reveal the reaction dynamics and the degree of thermalization attained. Enhancement of strangeness and/or antibaryons may signal the creation of the quark gluon plasma.

As an example of the importance of these measurements the expectations for the "baryon free" region for Au+Au central collisions at RHIC energies are compared for two different calculations, both of which are based in part on theoretical string models and in part on scaling from $p+p$ data, particularly from the CERN ISR $(\sqrt{s}=64 \mathrm{GeV})$. Plotted in Fig. VII. 1 is the rapidity density for the net baryon number, namely the multiplicity of $p-\bar{p}+n-\bar{n}$ after all

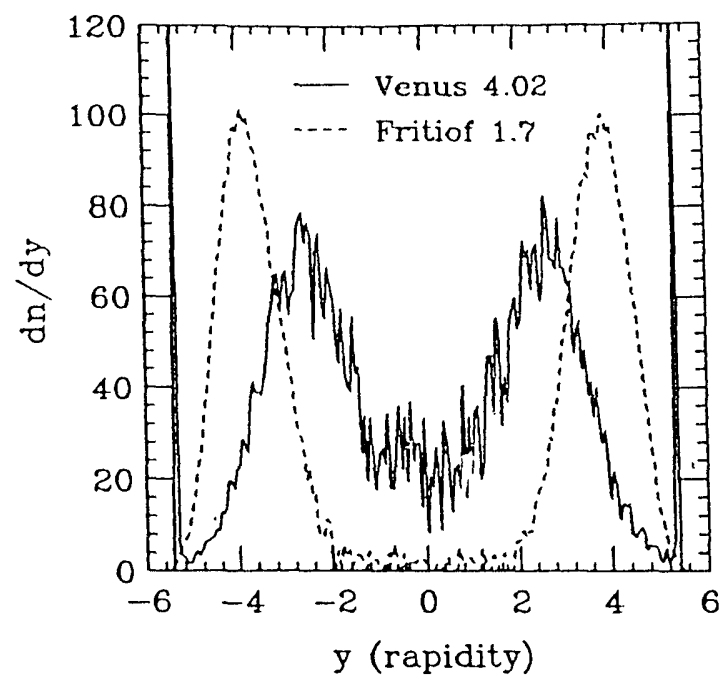

Fig. VII.1 Net baryons from central Au+Au Collisions

unstable particles have decayed. The FRITIOF 1.7 model, which describes the $p+p$ data of the 
ISR quite well, predicts a flat, baryon poor region in midrapidity for central Au+Au collisions at RHIC, and peaks at $y= \pm 3.8$, shifted 1.6 units from the beam rapidity of \pm 5.4 . The prediction of the VENUS 4.02 code for the same system is dramatically different from FRITIOF, showing no baryon free region, and smaller peaks at $y= \pm 2.6$. Thus the increased "stopping" inherent in VENUS has spread the colliding baryons over the whole rapidity region. Recent results from the RQMD (Relativistic Quantum Molecular Dynamics) transport model, which also incorporates a string breaking scheme, support the VENUS result, namely that a baryon free, boost invariant region is not obtained at $\mathrm{RHIC}$ energies.

This discussion has shown the importance of actually measuring the rapidity dependence of the baryon yields, but equally strong arguments can be made for measuring baryon and meson $p_{t}$ distributions over as wide a range of rapidity as possible. Furthermore, it is important to systematically study these distributions as a function of the mass of the projectics as well as the violence (impact parameter) of the collision. Such information is critically needed to establish constraints on the theoretical models and for understanding the basic physical conditions for the quark gluon physics at RHIC.

With these goals in mind, the BRAHMS detector, which consists of both a magnetic forward angle spectrometer and a midrapidity spectrometer, has been designed to measure inclusive and semi--inclusive $\mathrm{p}^{ \pm}, \pi^{ \pm}$, and $\mathrm{K}^{ \pm}$spectra in the pseudorapidity interval $0 \leq \eta \leq 4\left(2.1^{\circ}\right.$ $\left.\leq \theta_{\text {la }} \leq \leq 90^{\circ}\right)$. In addition to the broad rapidity coverage, the spectrometers are also unique within the suite of RHIC detectors in their $p_{t}$ coverage for well identified particles: up to $p_{t}=1.5$ $\mathrm{GeV} / c$ at all pseudorapdities and up 10 at least $2.5 \mathrm{GeV} / \mathrm{c}$ for $\eta<3$. In addition, the centrality of the collisions is measured by a global multiplicity detector.

Fig. VII.2 shows a schematic floor plan of the spectrometers, which are both freely rotating. The midrapidity arm covers angles $30^{\circ} \leq \theta / a b \leq 90^{\circ}$, while the forward arm coverage is

D4

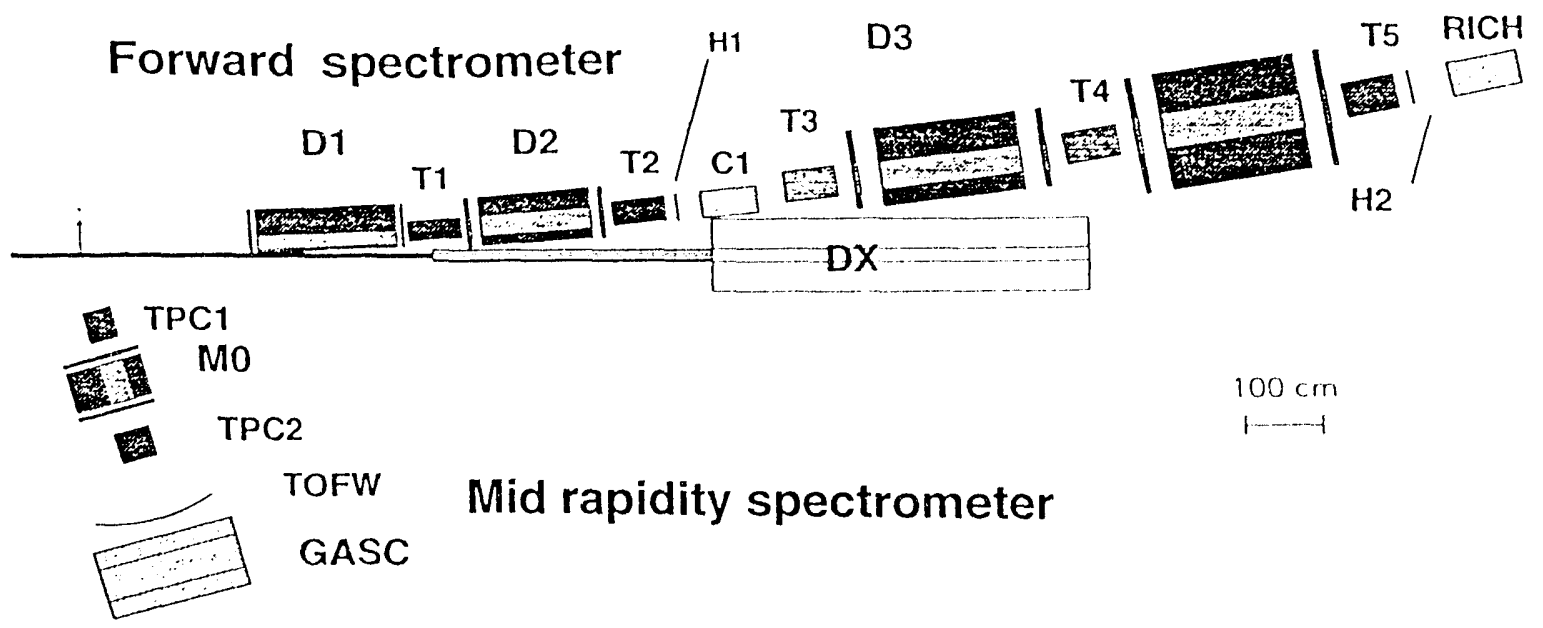

Fig. VII.2 Schematic floor plan of the BRAHMS detector. 
in two parts: the full forward spectrometer covers $2.1^{\circ}-15^{\circ}$ and the front section DI through $\mathrm{C} 2$ swings loose to cover $15^{\circ}-30^{\circ}$. The multiplicity array and the beam-beam counters are not shown.

The forward spectrometer arm will contain four magnets (D1--D4) for sweeping and analyzing primary particles emerging from the reaction. In order to fit in the space by the beam pipe at the most forward setting of $2.1^{\circ}, \mathrm{Dl}$ is of a septum design; in order to bend $25 \mathrm{GeV} / \mathrm{c}$ particles, it will have a field of up to 1.2 T. The other magnets, D2--D4 and M0 on the midrapidity arm, are of conventional design.

The forward arm tracking elements $\mathrm{T} 1$ and $\mathrm{T} 2$ each consist of a small time projection chamber (TPC), which provides good three dimensional track identification and rejection of background, sandwiched by projective drift chambers on both sides to provide precise spatial information. Such a scheme has already been developed for Experiment 866 at the AGS, where the particle densities of order $0.1 \mathrm{~cm}^{-2}$ are similar to those expected for detectors $\mathrm{T} 1$ and $\mathrm{T} 2$ at RHIC. Tracking detectors T3--T5 will be conventional drift chambers.

The lower momentum part of the particle identification for the forward arm is based on two time-of-flight hodoscopes, $\mathrm{H1}$--2. With an anticipated time resolution better than $100 \mathrm{ps}$, the $\mathrm{Hl}$ array at $9 \mathrm{~m}$ from the vertex will provide $4 \sigma$ separation of $\pi$ and $\mathrm{K}$ to $3 \mathrm{GeV} / \mathrm{c}$ and $\mathrm{K} / \mathrm{p}$ to 5.2 $\mathrm{GeV} / c$. The $\mathrm{H} 2$ array (after D4) will have $\pi / \mathrm{K}$ separation to $4.3 \mathrm{GeV} / c$ and $\mathrm{K} / \mathrm{p}$ to $7.2 \mathrm{GeV} / c$.

High momentum particles will be identified at two places in the forward spectrometer: namely after D2, covering the range of 2.5--6 GeV/c, and behind D4 with coverage from 4 to 25 GeV/c. The first region behind D2 will be instrumented by the use of a conventional segmented Cerenkov tank, $\mathrm{Cl}$. A ring-imaging Cerenkov detector ( $\mathrm{RICH}$ ) will sit behind $\mathrm{H} 2$ to perform $\mathrm{K} / \pi$ separation up to $\approx 20 \mathrm{GeV} / \mathrm{c}$ and $\mathrm{K} / \mathrm{p}$ separation much higher. This is accomplished by determining the ring radius with an accuracy of $\Delta R / R \sim 2 \%$.

The midrapidity arm is a single dipole magnetic spectrometer of $8 \mathrm{msr}$ solid angle designed to cover the angular region from $30^{\circ}$ up to $90^{\circ}$ and to measure and identify particles with momenta in the range of $p \sim 0.2 \mathrm{GeV} / \mathrm{c}$ to $5 \mathrm{GeV} / \mathrm{c}$. Since many particles detected behind the magnet $\mathrm{M}()$ arise from decays and secondaries, it is necessary include tracking in front of the magnet. This purpose will be served by a the time-projection chambers TPC1 and TPC2. The time-of-flight detection will be done with an array of 250 plastic scintillators placed at $4 \mathrm{~m}$. Each element will use the same kind of tubes and electronics as used in the $\mathrm{H} 1$ and $\mathrm{H} 2$ hodoscopes in the forward arm. This will give $\pi / \mathrm{K}$ separation up to $2 \mathrm{GeV} / \mathrm{c}$ and $\mathrm{K} / \mathrm{p}$ to about $3.5 \mathrm{GeV} / \mathrm{c}$. A pressurized gas Cerenkov threshold detector with segmentation $\approx 15$ will give $\pi / \mathrm{K}$ separation up to $\approx 6 \mathrm{GeV} / \mathrm{c}$. This will be augmented with a suitable back counter to measure $\mathrm{K}$ conversions in the Cerenkov tank.

A charged-particle multiplicity counter with nominal pseudorapidity coverage $|\eta| \leq 3.6$ 
will provide a global trigger, determining the degree of centrality of the event. An array of beam-beam counters at $6 \mathrm{~m}$ backwards and forwards of the beam vertex will provide a start-time for the time-of-flight counters and will determine the vertex position to roughly $1 \mathrm{~cm}$. The beam-beam counters will be phototubes with Cerenkov radiators in front.

The two spectrometers will be used with all beams and energies available at RHIC and will be ready when RHIC turns on. This is one of the "small" RHIC experiments and has a collaboration of 30 physicists from BNL, CRN-Strasbourg, the Chinese Institute of Atomic Energy in Beijing, New York University, Texas A. \& M., and U.C. Berkeley Space Sciences Laboratory.

\section{Suggested Further Reading}

D. Beavis, et al, Letter of intent for a forward angle and midrapidity hadron spectrometer at RHIC, RHIC-LOI-8, Brookhaven National Laboratory (1992).

B. Andersson, et. al., Nucl. Phys. B281, 289 (1987) [FRITIOF Model].

K. Werner, Z. Physics C42, 85 (1989) [VENUS Model]

Th. Schonfeld, et. al., Nucl. Phys. A544, 439c (1992) [RQMD calculations]. 

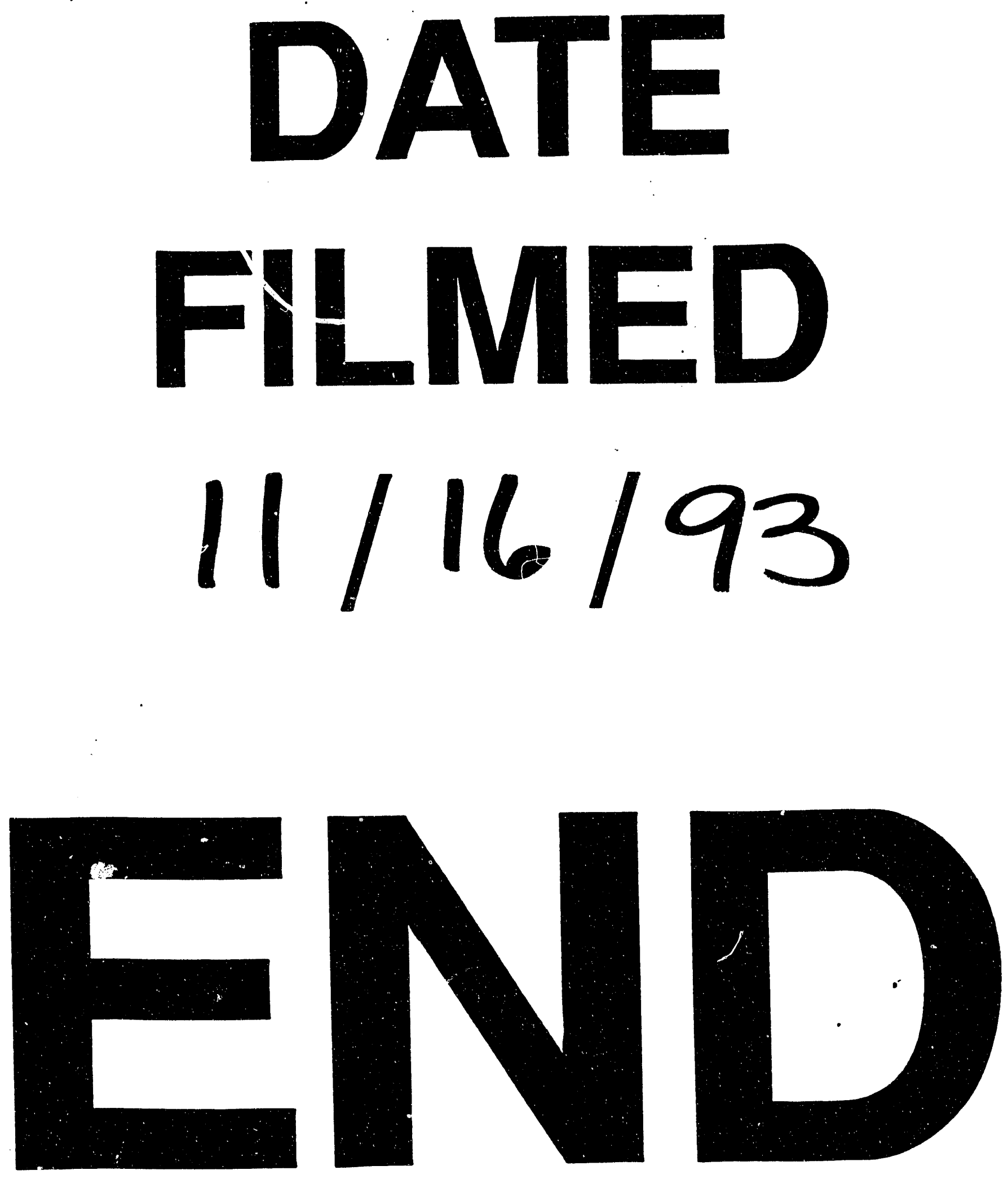
